TAN-FNT-96/003

hep-th/9612129

\title{
Covariant Quantization of the Skyrmion
}

\author{
M. Kruczenski i \\ Departamento de Física, Comisión Nacional de Energía Atómica, Av. del \\ Libertador 8250, 1429 Buenos Aires, Argentina.
}

and

L.E. Oxman日

Instituto de Fisica, Universidade Federal do Rio de Janeiro, C.P. 68528, Rio de Janeiro, Brasil.

\begin{abstract}
We obtain the internal degrees of freedom of the skyrmion (spin and isospin) within a manifestly Lorentz covariant quantization framework based on defining Green functions for skyrmions and then, the S-matrix via LSZ reduction. Our method follows Fröhlich and Marchetti's definition of Euclidean soliton Green functions, supplemented with a careful treatment of the boundary conditions around the singularities. The covariant two-point function obtained propagates a tower of spin equal to isospin particles. Our treatment contains the usual method of collective coordinates, as a non-relativistic limit and, because of the new topology introduced, it leads, in a natural way, to the inequivalent (boson/fermion) quantizations of the $\mathrm{SU}(2)$ skyrmion.
\end{abstract}

\section{Introduction}

Since the work of Witten [1,2], the Skyrme model [3] has received renewed attention as the low energy limit of QCD, as it is supposed to describe QCD

${ }^{1}$ E-mail: martink@tandar.cnea.edu.ar

${ }^{2}$ Partially supported by CLAF/CNPq, Brasil.

To appear in Physics Reports (1995) 
in the large number of colors $\left(N_{c}\right)$ limit. Recently, B. Moussallam [4] gave further support to the soliton picture showing that the low energy expansion of chiral perturbation theory [5] supplemented with a large $N_{c}$ analysis is compatible with a stable soliton.

The large $N_{c}$ limit can be thought of as a semiclassical expansion, as $N_{c}$ appears in the combination $\hbar / N_{c}$ [6]. One starts from a minimum of the potential which is the classical soliton profile. The soliton solution has six zero modes corresponding to translations and rotations (or isorotations). Quantization of these zero modes gives rise to linear momentum, spin and isospin. An analysis of the wave functions [1] reveals the existence of a tower of particles with spin equal to isospin.

Also, as the mass of the skyrmion scales as $N_{c}$, in the large $N_{c}$ limit a nonrelativistic treatment of the skyrmion is justified. Although this is correct in principle, it is not always desirable to break Lorentz invariance as one looses the possibility of applying standard knowledge from quantum field theory. For example, in reference [7], the equivalence to a relativistic field theory was advocated as an important point to solve the so called Yukawa problem. Therefore it is useful to introduce a manifestly Lorentz covariant quantization, defining Green functions for skyrmion fields. We believe that it is conceptually better to have a definition for these Green functions and, a posteriori, to take the non-relativistic limit.

Fröhlich and Marchetti [8] introduced Euclidean Green functions for skyrmions following previous developments on soliton quantization [9, 10. In reference [11] similar ideas were considered in the context of gauge theories.

In the present paper we will consider the covariant approach of ref. [8] but, in order to obtain the skyrmion internal degrees of freedom (spin and isospin), we will prescribe the boundary conditions which the field satisfies around the singularities. This treatment will allow us to relate the covariant quantization approach to more traditional ones for skyrmion quantization円. Also, this covariant quantization is a natural candidate for the skyrmion quantum field theory underlying the work of N. Dorey, J. Hughes and M.P. Mattis [7].

In section 2 the Green functions defined in [8] are reviewed. In section 3 the boundary conditions are discussed, showing that they bring in new topology. In section 6 the propagator is shown to transform correctly under Lorentz and isospin transformations. Further arguments are given in section 5 to show that the propagator describes a tower of spin equal to isospin particles. In section 6 we give the LSZ rules to construct the S-matrix and discuss the relation of our calculation to the computation of ref. [7]. In section 7] our conclusions are given. Finally, in an appendix, we summarize the definition of arbitrary spin

1 For a review see [12] 
propagators due to Weinberg [13].

\section{Green functions}

The Skyrme model is defined by the following Lagrangian

$$
\mathcal{L}=\frac{f_{\pi}^{2}}{4} \operatorname{Tr} \partial_{\mu} U^{\dagger} \partial^{\mu} U+\frac{1}{32 e^{2}} \operatorname{Tr}\left(\left[U^{\dagger} \partial_{\mu} U, U^{\dagger} \partial_{\nu} U\right]^{2}\right)
$$

where $U$ is a matrix belonging to $\mathrm{SU}(2)$. The lagrangian is invariant under global $\mathrm{SU}(2)_{\mathrm{L}} \times \mathrm{SU}(2)_{\mathrm{R}}$ chiral symmetry transformations $U \rightarrow g_{L} U g_{R}^{\dagger}, g_{L}, g_{R} \in$ $\mathrm{SU}(2)$. This symmetry is spontaneously broken. If one chooses the vacuum expectation value of $U$ to be $\langle U\rangle=1$, the surviving symmetry is the diagonal subgroup $\mathrm{SU}(2)_{\mathrm{V}}\left(g_{L}=g_{R}\right)$. A convenient parametrization of $U$ is

$$
U=\exp \left(\mathrm{i} \phi_{\mathrm{a}} \tau^{\mathrm{a}}\right)
$$

where $\tau^{a}$ are the Pauli matrices, and the fields $\phi_{a}$ describe the pions which have zero vacuum expectation value $\left(\langle U\rangle=1 \Rightarrow\left\langle\phi_{a}\right\rangle=0\right)$. One can also consider a pion mass term and higher derivative terms such as $-\epsilon_{6} B^{\mu} B^{\mu}$, where $\epsilon_{6}$ is a constant and $B^{\mu}$ is defined in eq. (3) below. From now on, we will work in Euclidean space and will use upper indices $(\mu=1, \ldots, 4)$ to denote space-time components. In the following, 4-dimensional Euclidean rotations will be called Lorentz transformations for the sake of brevity and to distinguish them from 3 -dimensional rotations. However this is an abuse of language and the reader should keep in mind that we are working in Euclidean space-time.

Static configurations with finite energy must satisfy $U \rightarrow 1$ at infinity, this amounts to compactify space to $S^{3}$. Thus, a static configuration defines a map $S^{3} \rightarrow \mathrm{SU}(2)$, that is, an element of $\Pi_{3}\left(S^{3}\right)=Z$. The baryonic number $B$, defined as the integral of the topological current

$$
\begin{aligned}
B^{\mu} & =\frac{\epsilon^{\mu \nu \alpha \beta}}{24 \pi^{2}} \operatorname{Tr}\left[\left(U^{\dagger} \partial^{\nu} U\right)\left(U^{\dagger} \partial^{\alpha} U\right)\left(U^{\dagger} \partial^{\beta} U\right)\right], \\
B & =\int d^{3} x B^{4}
\end{aligned}
$$

is the winding number associated with $\Pi_{3}\left(S^{3}\right)$ and therefore is conserved. Configurations with $B=1$ describe nucleon states. The energy of the $B=1$ static configuration that minimizes the action is concentrated in space; then, this configuration can be approximately treated as a rigid body rotating in space and also in internal space. Spin and isospin arise quantifying these collective degrees of freedom [罒]. 
If there are quantum states associated with the classical soliton then it should be possible to define Green functions such as

$$
G^{n_{1} \ldots n_{p}, a_{1} \ldots a_{r}}\left(x_{1} \ldots x_{p}, y_{1} \ldots y_{r}\right)=\left\langle T\left(\psi^{n_{1}}\left(x_{1}\right) \ldots \psi^{n_{p}}\left(x_{p}\right) \phi_{a_{1}}\left(y_{1}\right) \ldots \phi_{a_{r}}\left(y_{r}\right)\right)\right\rangle,
$$

where $\psi^{n_{i}}\left(x_{i}\right)$ (formally) represents an operator destroying a soliton of winding number $n_{i}$ at $x_{i}$ or creating an antisoliton $-n_{i}$ at $x_{i}$.

Following [8] we introduce a path integral over fields defined on space-time compactified to $S^{4}$ and with the points $x_{1} \ldots x_{p}$ removed $\left(M_{1 \ldots p}=\left(R^{4} \cup\right.\right.$ $\left.\{\infty\}) \backslash\left\{x_{1}, \ldots, x_{p}\right\}\right)$. The manifold $M_{1 \ldots p}$ is contractible to a bundle of $p-1$ spheres $S^{3}$ with one common point, implying that maps from $M_{1 \ldots p}$ to $\mathrm{SU}(2)$ are classified by $p-1$ independent integers. These integers can be written in terms of $p$ integers $n_{i}$, satisfying $n_{1}+\cdots+n_{p}=0$, defined by

$$
n_{i}=\int_{S_{i}} \mathrm{dS} \mathrm{n}^{\mu} \mathrm{B}^{\mu}, \mathrm{i}=1 \ldots \mathrm{p}
$$

where $S_{i}$ is a three sphere enclosing $x_{i}$ which does not enclose any other point $x_{j}(j \neq i), n^{\mu}$ is the normal to $S_{i}$ and $\mathrm{dS}$ is the surface element on $S_{i}$. Due to the fact that $U$ is constant at infinity, we have $n_{1}+\cdots+n_{p}=0$.

The skyrmion Green functions in eq. (田) were defined by Fröhlich and Marchetti in ref. [8]. Including the pion field definition (21), the mixed pion-skyrmion Green functions read

$$
G^{n_{1} \ldots n_{p}, a_{1} \ldots a_{r}}\left(x_{1}, \ldots, x_{p}, y_{1}, \ldots y_{r}\right)=\int \mathcal{D}[U] \phi_{a_{1}}\left(y_{1}\right) \ldots \phi_{a_{r}}\left(y_{r}\right) \exp \left(-\int_{M} \mathcal{L}[U]\right)
$$

(see [8] for an interpretation in terms of open line defects). $U=\exp \left(i \phi_{\mathrm{a}} \tau^{\mathrm{a}}\right)$ takes the value $U=1$ at space-time infinity and has topological number $n_{i}$ around $x_{i}(i=1, \ldots, p)$. A rigorous definition of the path integral would involve first defining it on a lattice and then letting the lattice approach the continuum [9]. Alternatively we can define the path integral by its perturbative expansion around a saddle point. In both cases, a finite ultraviolet cut-off $\Lambda$ will be required since the Skyrme model is non-renormalizable. In the case of chiral perturbation theory the limit $\Lambda \rightarrow \infty$ can be taken by absorbing the infinities in the higher order derivative terms, which are neglected at large distances.

In order to clarify the meaning of eq. (6) let us consider the soliton propagator

$$
G^{-1,+1}\left(x_{1}, x_{2}\right)=\int \mathcal{D}[U] \exp \left(-\int_{M_{12}} \mathcal{L}[U]\right)
$$

The path integral is performed over fields $U$ which have winding number +1 around $x_{2}$ and -1 around $x_{1}$. The situation is depicted in figure 1 . 


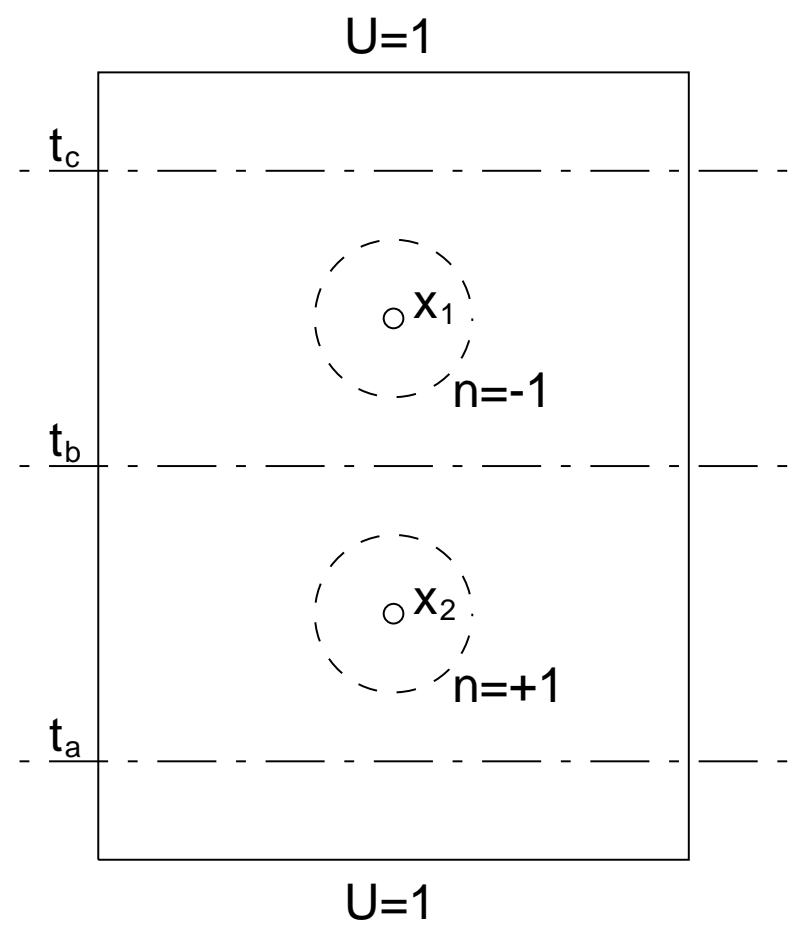

Fig. 1. To define the propagator, configurations over $R^{4} \backslash\left\{x_{1}, x_{2}\right\}$ are considered. See the discussion in the text.

Consider a spatial section at constant time $t=t_{a}$. By closing this section at $t=-\infty$ we see that it has topological number 0 , that is, there are no topological excitations at $t=t_{a}$. The same argument for a section at $t=t_{b}$ shows that it has winding number +1 and, at $t=t_{c}$, the winding number is again 0 . Then, we see that a $B=1$ soliton is created at $x_{2}$ and destroyed at $x_{1}$. Furthermore, if we look at the time inverted process, we find that section $t=t_{b}$ has topological number -1 , because definition (3) picks up a minus sign when the normal is reversed. Thus, the figure also describes an antisoliton propagating backwards in time, that is, at $x_{2}$ a soliton is created or equivalently an antisoliton destroyed, the effect expected from a quantum field operator associated with the antisoliton. Remember that a field $\psi$ destroys the particle and creates the antiparticle whereas $\psi^{\dagger}$ does the opposite.

The following step is to find a saddle point configuration for our path integral. This can be done proposing a (time-dependent) Skyrme ansatz

$$
U=\exp \left(\operatorname{if}(\mathrm{u}, \mathrm{v}) \breve{\mathrm{x}}_{\mathrm{a}} \tau^{\mathrm{a}}\right)
$$

where $\breve{x}_{a}=x_{a} / r, r=\sqrt{x_{1}^{2}+x_{2}^{2}+x_{3}^{2}}$, and $u=e f_{\pi} r, v=e f_{\pi} t$ are adimen- 
sional. Minimizing the action, gives an equation for $f(u, v)$ :

$$
\begin{aligned}
\left(2 \sin ^{2} f+u^{2}\right)\left(\frac{d^{2} f}{d v^{2}}+\frac{d^{2} f}{d u^{2}}\right)+\sin (2 f)\left(\left(\frac{d f}{d v}\right)^{2}+\left(\frac{d f}{d u}\right)^{2}\right)+ \\
+2 u \frac{d f}{d u}-\sin (2 f)\left(1+\frac{\sin ^{2} f}{u^{2}}\right)=0 .
\end{aligned}
$$

The boundary condition $U=1$ at space-time infinity requires that $f(u, v) \rightarrow 0$ when $v \rightarrow \pm \infty$ or $u \rightarrow \infty$. At $u=0$, for $U$ to be single valued, $f$ must be 0 or $\pi$. Continuity of $f$ (on the manifold $M_{12}$ ) and the requirement that $U$ must have topological number 1 around $x_{2}$ and -1 around $x_{1}$ leads to

$$
\begin{array}{ll}
f(u, v)=0 \quad \text { if } \quad v \rightarrow \pm \infty \text { or } u \rightarrow \infty, \\
f(0, v)=0 \quad \text { if } v<e f_{\pi} x_{2}^{4} \text { or } v>e f_{\pi} x_{1}^{4}, \\
f(0, v)=\pi \quad \text { if } e f_{\pi} x_{2}^{4}<v<e f_{\pi} x_{1}^{4} .
\end{array}
$$

These boundary conditions respect the cylindrical symmetry of the ansatz. In the next section we will discuss a more general situation.

The numerical solution to eqs. (9) and (10) is depicted in figure 2 where we see that $f$ (and so the fields $\phi_{a}$ ) is approximately zero before $x_{2}^{4}$ or after $x_{1}^{4}$ and is almost equal to the static soliton profile in between. This supports the picture that a skyrmion is created at $x_{2}$ and propagates towards $x_{1}$ where it is destroyed. Furthermore, since the solution is almost time independent in the time interval $\left(x_{2}^{4}, x_{1}^{4}\right)$ and equal to the static profile, the action is approximately given by $S=M_{\mathrm{cl}}\left|x_{2}-x_{1}\right|$, where $M_{\mathrm{cl}}$ is the energy of the static solution. Hence, the saddle point approximation to the propagator contains a factor $\exp \left(-M_{\mathrm{cl}}\left|x_{2}-x_{1}\right|\right)$ which is the exponential behavior of the Euclidean propagator for a particle with mass $M_{\mathrm{cl}}$. In section 5 we will improve this approximation, obtaining the correct, spin equal to isospin, particle spectrum.

Let us now consider spheres $S_{R}$ of radius $R<\left|x_{2}-x_{1}\right|$ around $x_{2}$. All these spheres have topological number 1 but the topological density, which in this case is given by $\rho\left(x_{\mu}\right)=B^{\mu}\left(x-x_{2}\right)^{\mu} /\left|x-x_{2}\right|$, will be differently distributed. Replacing the ansatz of eq. (8) in the last expression it follows that the density is only a function of $\theta$, the angle between $x-x_{2}$ and the 4-axis, and is given by

$$
\rho(\theta)=-\frac{1}{2 \pi^{2}} \frac{\sin ^{2}(f) \partial_{\theta} f}{\sin ^{2}(\theta)} .
$$

The numerical solution $f(u, v)$ as a function of $\theta$ can be obtained, by interpolation, from the formula

$$
f(\theta)=f\left(R \sin (\theta), e f_{\pi} x_{2}^{4}+R \cos (\theta)\right) .
$$

The result for several values of $R$ (in units of $\left(e f_{\pi}\right)^{-1}$ ) is depicted in figure 3 . 


This figure is available at
http://www.tandar.cnea.edu.ar/preprints/FNT/96/003.tex

Fig. 2. Numerical solution for $f(u, v)$ that minimizes the action $\left(u=e f_{\pi} r, v=e f_{\pi} t\right)$. In the figure, $\left|x_{2}-x_{1}\right|=6\left(e f_{\pi}\right)^{-1}$.

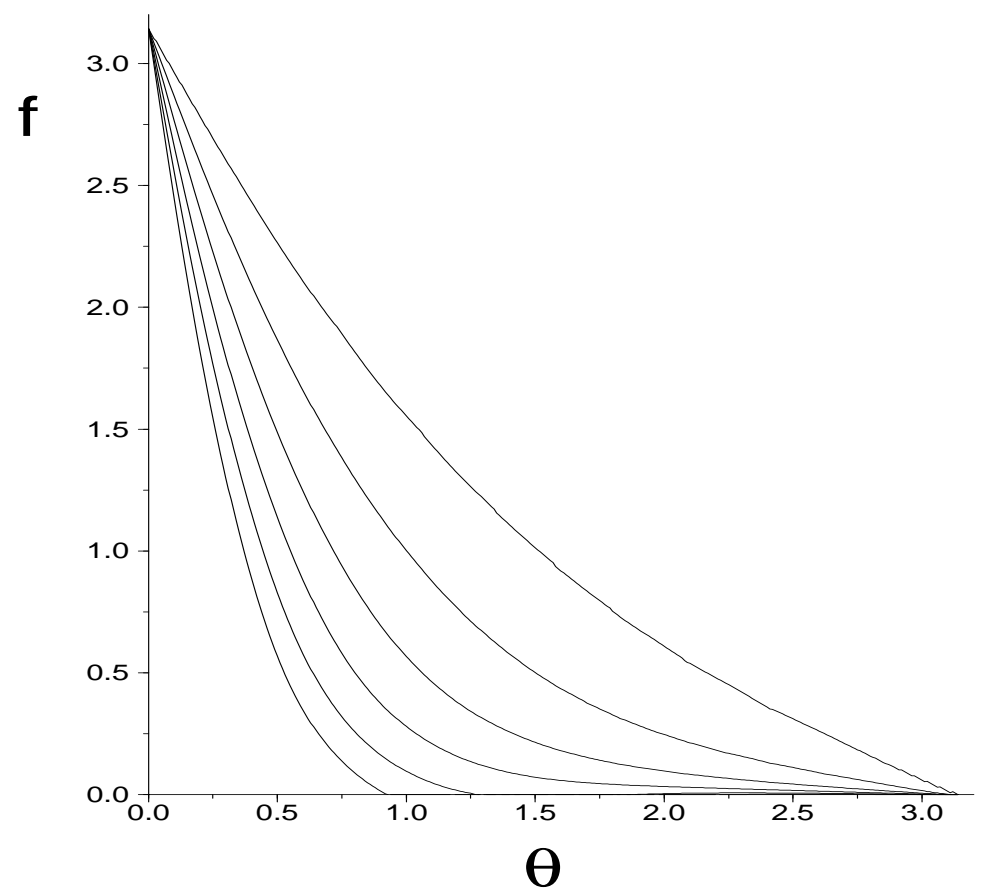

Fig. 3. $f$ as a function of $\theta$ for different radius, corresponding (from the upper to the lower curve) to $R=0.5,1.0,1.5,2.0,2.5,3.0$ in units of $\left(e f_{\pi}\right)^{-1}$, in the case $\left|x_{2}-x_{1}\right|=6\left(e f_{\pi}\right)^{-1}$.

In this figure, we see that near the singularity $(R \rightarrow 0) f(\theta)$ tends to $\pi-\theta$, which gives a uniform topological density (cf. eq. (11)). As one gets further from the singularity the topological density concentrates around the North pole of the sphere giving rise to a soliton moving towards $x_{1}$. This is again the graphical picture corresponding to a soliton appearing around $x_{2}$. This behavior of the solution (unlocalization for small $R$, and localization for large 
$R$ ) is in agreement with studies of the static ansatz on a sphere [14 and is in correspondence with the fact that at short distances the chiral symmetry is unbroken whereas it is broken at large distances.

We end this section noticing that the behavior $f(\theta) \approx \pi-\theta$ near $x_{2}$ is equivalent to

$$
U(x) \approx-X_{2}^{\dagger}(x), \quad X_{2}(x)=\frac{x^{4}-x_{2}^{4}}{\left|x-x_{2}\right|}+\mathrm{i} \frac{\mathrm{x}^{\mathrm{a}}-\mathrm{x}_{2}^{\mathrm{a}}}{\left|\mathrm{x}-\mathrm{x}_{2}\right|} \tau_{\mathrm{a}},
$$

where $\tau_{a}$ are the Pauli matrices. A similar analysis around $x_{1}$, reveals that $U$ behaves as $X_{1}$, where $X_{1}$ is defined as $X_{2}$, but with $x_{2}$ replaced by $x_{1}$. These behaviors will be useful in the next section, when discussing the boundary conditions to be imposed on the fields.

\section{The boundary conditions}

The numerical saddle point obtained in the previous section is not invariant under rotations or isorotations, that is, there are several other saddle points or equivalently, several classical trajectories. This is due to the fact that we have not yet specified the boundary conditions satisfied by the field around $x_{1}$ and $x_{2}$.

In order to do so, we extract two small balls $B_{1}^{4}$ and $B_{2}^{4}$ of radius $\epsilon \rightarrow 0$ around $x_{1}$ and $x_{2}$ respectively. Then, we need to specify the values taken by the field $U$ on the surfaces $S_{1}^{3}=\partial B_{1}^{4}$ and $S_{2}^{3}=\partial B_{2}^{4}$ of these balls, satisfying the condition that $U$ must have topological number +1 around $x_{2}$ and -1 around $x_{1}$.

In principle, any function $U: S^{3} \rightarrow \mathrm{SU}(2)$ can be chosen, but we will restrict ourselves to a minimum set of boundary conditions which close under Lorentz and isospin transformations. At the end of section 2 we saw that near $x_{2}$ the numerical solution behaves as $-X_{2}^{\dagger}$, resulting in a uniform topological density. Performing Lorentz transformations we obtain, around the singularity, the behaviors $S_{1}\left(-X_{2}\right)^{\dagger} S_{2}^{\dagger}\left(S_{1}, S_{2} \in \mathrm{SU}(2)\right.$, see eq. (29) below) without altering the uniform topological density. This suggest that all the boundary conditions $S_{1}\left(-X_{2}\right)^{\dagger} S_{2}^{\dagger}$ should be treated on an equal footing to obtain a representation of Lorentz and isospin symmetries. Thus, the boundary conditions are taken to be

$$
\begin{aligned}
& \left.U\right|_{S_{1}^{3}}=A_{1} X_{1} B_{1}^{\dagger}, \\
& \left.U\right|_{S_{2}^{3}}=A_{2}\left(-X_{2}\right)^{\dagger} B_{2}^{\dagger},
\end{aligned}
$$

where $A_{1}, B_{1}, A_{2}, B_{2}$ belong to $\mathrm{SU}(2)$ and $X_{1}, X_{2}$ are the $\mathrm{SU}(2)$ matrices de- 
fined by

$$
X_{1,2}(x)=\frac{x^{4}-x_{1,2}^{4}}{\left|x-x_{1,2}\right|}+\mathrm{i} \frac{\mathrm{x}^{\mathrm{a}}-\mathrm{x}_{1,2}^{\mathrm{a}}}{\left|\mathrm{x}-\mathrm{x}_{1,2}\right|} \tau_{\mathrm{a}},
$$

As an aside remark, note that, in terms of the vector field $L_{\mu}=U^{\dagger} \partial_{\mu} U$, these conditions look like the insertion of a zero-radius instanton (anti-instanton) at $x_{1}\left(x_{2}\right) \cdot{ }^{2}$

Each pair $A_{i}, B_{i}$ determines a map $S_{i}^{3} \rightarrow \mathrm{SU}(2)$. In fact, as exactly the same map is determined by $-A_{i},-B_{i}$, the boundary condition is defined by a point in the manifold $\mathrm{SU}(2) \times \mathrm{SU}(2) / \mathrm{Z}_{2}$. These boundary conditions represent the internal degrees of freedom of the skyrmion fields.

Given two points $p_{1}$ and $p_{2}$ on $\mathrm{SU}(2) \times \mathrm{SU}(2) / \mathrm{Z}_{2}$ we define a propagator

$$
G\left(x_{1}, p_{1} ; x_{2}, p_{2}\right)=\lim _{\epsilon \rightarrow 0} \int \mathcal{D}[U] \exp \left(-\int_{M_{12}} \mathcal{L}[U]+2 C_{\epsilon}\right)
$$

where $U$ at $S_{1}^{3}$ is given by $p_{1}$ and at $S_{2}^{3}$ by $p_{2}$, according to eq. (14) (at spacetime infinity we take $U=1$, the vacuum configuration $) . C_{\epsilon}=-\left(3 \pi^{2} / e^{2}\right) \log (\epsilon)$ is added to cancel the infinite contribution to the action due to the singularity and can be thought of as wave function renormalization of the skyrmion fields. Including higher order derivative terms in the action would produce worse singularities and $C_{\epsilon}$ should be properly redefined; the inclusion of, say, the sixth order term $-\epsilon_{6} B^{\mu} B_{\mu}$ requires that $C_{\epsilon}=-\left(6 \pi^{2} / e^{2}\right) \log (\epsilon)-\epsilon_{6}\left(2 \pi^{2} \epsilon^{2}\right)^{-1}$. Of course, at short distances, the most significant terms are precisely those neglected at large distances, reflecting the fact that near the singularities the lagrangian in eq. (11) is not adequate. However this does not modify the symmetry arguments and, far from the singularities, the classical solution is insensitive to the higher order terms.

Now, we will show that the imposed boundary conditions lead to a natural discussion of the different possible quantizations of the Skyrme model. Up to now, as $M_{12}=\left(R^{4} \cup\{\infty\}\right) \backslash\left\{x_{1}, x_{2}\right\}$ is contractible to $S^{3}$, the configurations $U: M_{12} \rightarrow \mathrm{SU}(2)$ are classified by an integer. However, when we fix the initial and final points we have a map

$$
U: S^{3} \times[0,1] \rightarrow \mathrm{SU}(2)
$$

because $M_{12}$ can be deformed to a cylinder with base $S^{3}$. Here, $\partial B_{1}^{4}$ and $\partial B_{2}^{4}$ are the bases of the cylinder and the parameter in $[0,1]$ represents the continuous deformation of $\partial B_{2}^{4}$ into $\partial B_{1}^{4}$, passing through sections between $x_{1}$ and $x_{2}$ (remember that space-time infinity is identified). Two such maps are

2 In our case we do not have a gauge theory, but one can define the Green functions in terms of a field $U$ coupled to a singular $L_{\mu}$ as described in 8 
not necessarily homotopic. The situation is similar to that of a plane with a hole. Paths with free end points are all homotopic but paths with fixed end points are classified by $\Pi_{1}\left(S^{1}\right)=Z$.

In our case suppose we give two configurations

$$
U_{1}, U_{2}: S^{3} \times[0,1] \rightarrow \mathrm{SU}(2),
$$

which satisfy the same boundary conditions at 0 and 1 . They can be used to define a map $U: S^{3} \times S^{1} \rightarrow \mathrm{SU}(2)$ as:

$$
U(X, t)= \begin{cases}U_{1}(X, 2 t) & \text { if } t \in[0,1 / 2] \\ U_{2}(X, 2(1-t)) & \text { if } t \in[1 / 2,1]\end{cases}
$$

It is easy to verify that $U$ is continuous at $1 / 2$ and periodic $(U(X, 0)=$ $U(X, 1))$, provided that $U_{1}$ and $U_{2}$ satisfy the same boundary conditions at $t=0,1$. This map is the analog of the closed path which can be constructed from two paths with the same end points, in the simple example of the plane with a hole.

Two configurations $U_{1}$ and $U_{2}$ are homotopic if the map $U: S^{3} \times S^{1} \rightarrow \mathrm{SU}(2)$ can be extended to a map $S^{3} \times D_{2} \rightarrow \mathrm{SU}(2)$, where $D_{2}$ is a two dimensional disc $\left(\partial D_{2}=S^{1}\right)$. In general, there is an obstruction to such an extension given by $\Pi_{4}(\mathrm{SU}(2))=\mathrm{Z}_{2}$ [2], this means that there are two different homotopy classes. A way of distinguishing them is to trivially embed $\mathrm{SU}(2)$ into $\mathrm{SU}(3)$ and evaluate the Wess-Zumino-Witten term. This gives 0 for the trivial class and $\pi$ for the non-trivial one. If we were considering $\mathrm{SU}(3)$ solitons all the paths would be homotopic and the Wess-Zumino-Witten term would give a different contribution for each path [2]. We would like to point out that the need of considering an extension of a map $U: S^{3} \times S^{1} \rightarrow \mathrm{SU}(2)$ to a map $U: S^{3} \times D_{2} \rightarrow \mathrm{SU}(2)$ to study the different possible inequivalent quantizations of the Skyrme model, which in [2] was proposed by imposing periodic boundary conditions to calculate the partition function, here also arises naturally from the Fröhlich and Marchetti's formalism supplemented with fixed boundary conditions around the singularities.

The path integral defining the propagator can then be split into two integrals, one over each homotopy class. A phase equal to 0 or $\pi$ can be assigned to the non-trivial class, which corresponds to adding or subtracting the two contributions:

$$
G\left(x_{1}, p_{1} ; x_{2}, p_{2}\right)=G^{(1)}\left(x_{1}, p_{1} ; x_{2}, p_{2}\right) \pm G^{(2)}\left(x_{1}, p_{1} ; x_{2}, p_{2}\right)
$$

The plus (minus) sign will correspond to quantizing the soliton as a boson (fermion) (see section 5). 
Now, suppose that we have a path in some topological class which ends at a boundary condition associated to $A_{2}, B_{2}$; if we adiabatically move this last boundary condition, leaving $A_{1}, B_{1}$ fixed, to get a boundary condition associated to $-A_{2},-B_{2}$ (which represents the same point as $A_{2}, B_{2}$ and $-A_{2},-B_{2}$ are identified), the new path will be in a different topological class. This is due to the fact that the new path can be obtained by composing the original path with a "closed" path, based on $\partial B_{2}^{4}$, that starts at $\left(A_{2}, B_{2}\right)$ and ends at $\left(-A_{2},-B_{2}\right)$. This last path is non-trivial and so the original path will change from homotopy class. Thus, changing adiabatically the final condition from $\left(A_{2}, B_{2}\right)$ to $\left(-A_{2},-B_{2}\right)$ interchanges $G^{(1)}$ and $G^{(2)}$.

This result allows us to continuously extend the boundary conditions from $\mathrm{SU}(2) \times \mathrm{SU}(2) / \mathrm{Z}_{2}$ to $\mathrm{SU}(2) \times \mathrm{SU}(2)$, defining a new function $\tilde{G}$ according to

$$
\begin{aligned}
\tilde{G}\left(x_{1}, A_{1}, B_{1} ; x_{2}, A_{2}, B_{2}\right) & =\tilde{G}\left(x_{1},-A_{1},-B_{1} ; x_{2},-A_{2},-B_{2}\right) \\
& =G^{(1)}\left(x_{1}, p_{1} ; x_{2}, p_{2}\right) \\
\tilde{G}\left(x_{1}, A_{1}, B_{1} ; x_{2},-A_{2},-B_{2}\right) & =\tilde{G}\left(x_{1},-A_{1},-B_{1} ; x_{2}, A_{2}, B_{2}\right) \\
& =G^{(2)}\left(x_{1}, p_{1} ; x_{2}, p_{2}\right)
\end{aligned}
$$

where $A_{1,2}, B_{1,2}$ and $-A_{1,2},-B_{1,2}$ both correspond to $p_{1,2}$ in $\mathrm{SU}(2) \times \mathrm{SU}(2) / \mathrm{Z}_{2}$ and we take the definitions to be valid when $A_{1}, A_{2}>0{ }^{3}$. In terms of $\tilde{G}$ the original propagator can be written as

$$
G\left(x_{1}, p_{1} ; x_{2}, p_{2}\right)=\tilde{G}\left(x_{1}, A_{1}, B_{1} ; x_{2}, A_{2}, B_{2}\right) \pm \tilde{G}\left(x_{1}, A_{1}, B_{1} ; x_{2},-A_{2},-B_{2}\right) .
$$

In the subsequent sections we will analyze the properties of $\tilde{G}$.

To close this section let us point out that the propagator $G$ can be associated with a field propagator

$$
G\left(x_{1}, p_{1} ; x_{2}, p_{2}\right)=\left\langle 0\left|T\left\{\psi_{A_{1}, B_{1}}^{1}\left(x_{1}\right) \psi_{A_{2}, B_{2}}^{-1}\left(x_{2}\right)\right\}\right| 0\right\rangle .
$$

Similarly, Green functions for any number $(p)$ of operators $\psi_{A, B}^{n}(x)$, and operators $\phi_{a}(y)$, can be defined (with the restriction $n_{i}= \pm 1$ ) according to:

$$
\begin{aligned}
& \left\langle 0\left|T\left\{\psi_{A_{1}, B_{1}}^{n_{1}}\left(x_{1}\right) \ldots \psi_{A_{p}, B_{p}}^{n_{p}}\left(x_{p}\right) \phi_{a_{1}}\left(y_{1}\right) \ldots \phi_{a_{r}}\left(y_{r}\right)\right\}\right| 0\right\rangle= \\
& \lim _{\epsilon \rightarrow 0} \int \mathcal{D}[U] \phi_{a_{1}}\left(y_{1}\right) \ldots \phi_{a_{r}}\left(y_{r}\right) \mathrm{e}^{-\mathrm{S}[\mathrm{U}]+\mathrm{pC}_{\epsilon}},
\end{aligned}
$$

together with the boundary conditions,

$$
\left.U\right|_{S_{i}}=\left\{\begin{array}{lr}
A_{i} X_{i} B_{i}^{\dagger} & \text { if } n_{i}=-1 \\
A_{i}\left(-X_{i}\right)^{\dagger} B_{i}^{\dagger} & \text { if } n_{i}=+1
\end{array}\right.
$$

${ }^{3}$ We define $A>0, A \in \mathrm{SU}(2)$ when $A=a_{0}+\mathrm{i} \overrightarrow{\mathrm{a}} \cdot \vec{\tau},\left(\mathrm{a}_{0}^{2}+\overrightarrow{\mathrm{a}}^{2}=1\right)$ is written with $a_{0}>0$. 


$$
X_{i}=\frac{x^{4}-x_{i}^{4}}{\left|x-x_{i}\right|}+\mathrm{i} \frac{\mathrm{x}^{\mathrm{a}}-\mathrm{x}_{\mathrm{i}}^{\mathrm{a}}}{\left|\mathrm{x}-\mathrm{x}_{\mathrm{i}}\right|} \tau_{\mathrm{a}} \in \mathrm{SU}(2),
$$

for small spheres $S_{i}$ around $x_{i}\left(C_{\epsilon}=-\left(3 \pi^{2} / e^{2}\right) \log (\epsilon)\right)$.

If $n>1$, the operator $\psi^{n}(x)$ could be defined as a suitable limit of $\psi^{1}\left(y_{1}\right) \ldots \psi^{1}\left(y_{n}\right)$ when $y_{i} \rightarrow x$. However we will not pursue this point here but instead, from now on we will consider only $\psi^{ \pm 1}$ fields which will be denoted as $\psi^{+1}=\psi$, $\psi^{-1}=\bar{\psi}$.

\section{Symmetries of the propagator}

The symmetries of the propagator can be found by performing different changes of variables, within the path integral, which are invariances of the action. In this way we learn how this symmetries act on the internal variables $A_{i}, B_{i}$.

Translations The change of variable $U(x) \rightarrow U(x+a)$ does not affect the action but affects the boundary conditions. Trivially, it follows that

$$
\tilde{G}\left(x_{1}+a, A_{1}, B_{1} ; x_{2}+a, A_{2}, B_{2}\right)=\tilde{G}\left(x_{1}, A_{1}, B_{1} ; x_{2}, A_{2}, B_{2}\right) .
$$

Isorotations With the change of variables $\tilde{U}=A U A^{\dagger},(A \in \mathrm{SU}(2))$ we obtain

$$
\begin{aligned}
S\left[A U A^{\dagger}\right] & =S[U] \\
A 1 A^{\dagger} & =1 \text { at } \infty \\
\left.\tilde{U}\right|_{S_{1}^{3}} & =\left.A U\right|_{S_{1}^{3}} A^{\dagger}=A A_{1} X_{1} B_{1}^{\dagger} A^{\dagger}=\left(A A_{1}\right) X_{1}\left(A B_{1}\right)^{\dagger} \\
\left.\tilde{U}\right|_{S_{2}^{3}} & =\left.A U\right|_{S_{2}^{3}} A^{\dagger}=A A_{2}\left(-X_{2}\right)^{\dagger} B_{2}^{\dagger} A^{\dagger}=\left(A A_{2}\right)\left(-X_{2}\right)^{\dagger}\left(A B_{2}\right)^{\dagger}(.27)
\end{aligned}
$$

Thus

$$
\tilde{G}\left(x_{1}, A A_{1}, A B_{1} ; x_{2}, A A_{2}, A B_{2}\right)=\tilde{G}\left(x_{1}, A_{1}, B_{1} ; x_{2}, A_{2}, B_{2}\right) .
$$

Lorentz transformations To describe particles of half-integer spin we must consider the universal covering of the Euclidean Lorentz group, namely $\mathrm{SU}(2) \times \mathrm{SU}(2)$. To each pair of matrices $S_{1}, S_{2} \in \mathrm{SU}(2)$ corresponds a matrix $\Lambda \in \mathrm{SO}(4)$. The transformation $x \rightarrow x^{\prime}=\Lambda x$ can be written in matrix form

$$
X \rightarrow X^{\prime}=S_{1} X S_{2}^{\dagger}, \quad X=\frac{x^{4}}{|x|}+\mathrm{i} \frac{\mathrm{x}^{\mathrm{a}}}{|\mathrm{x}|} \tau_{\mathrm{a}} \in \mathrm{SU}(2) .
$$

Now let us perform the change of variables

$$
U(x) \rightarrow \tilde{U}(x)=U\left(\Lambda^{-1} x\right)
$$


which leaves the action invariant. The boundary conditions transform as

$$
\begin{aligned}
& \left.\tilde{U}\left(X^{\prime}\right)\right|_{S_{1}^{\prime 3}}=\left.U(X)\right|_{S_{1}^{3}}=A_{1} X_{1} B_{1}^{\dagger}=A_{1} S_{1}^{\dagger} X_{1}^{\prime} S_{2} B_{1}^{\dagger}=\left(A_{1} S_{1}^{\dagger}\right) X_{1}\left(B_{1} S_{2}^{\dagger}\right)^{\dagger} \\
& \left.\tilde{U}\left(X^{\prime}\right)\right|_{S_{2}^{\prime 3}}=\left.U(X)\right|_{S_{2}^{3}}=A_{2}\left(-X_{2}^{\dagger}\right) B_{2}^{\dagger}=\left(A_{2} S_{2}^{\dagger}\right)\left(-X_{2}^{\prime \dagger}\right)\left(B_{2} S_{1}^{\dagger}\right)^{\dagger}
\end{aligned}
$$

which gives

$$
\tilde{G}\left(\Lambda x_{1}, A_{1} S_{1}^{\dagger}, B_{1} S_{2}^{\dagger} ; \Lambda x_{2}, A_{2} S_{2}^{\dagger}, B_{2} S_{1}^{\dagger}\right)=\tilde{G}\left(x_{1}, A_{1}, B_{1} ; x_{2}, A_{2}, B_{2}\right) .
$$

Parity The pion is pseudo-scalar, so under parity $U$ transforms as

$$
U\left(x^{4}, \vec{x}\right) \rightarrow \tilde{U}\left(x^{4}, \vec{x}\right)=U^{\dagger}\left(x^{4},-\vec{x}\right) .
$$

As a spatial reflection maps $X_{1,2} \rightarrow X_{1,2}^{\dagger}$, a parity transformation simply interchanges $A$ and $B$ in the boundary conditions. So, parity invariance of the action implies that

$$
\tilde{G}\left(x_{1}^{4}, \vec{x}_{1}, A_{1}, B_{1} ; x_{2}^{4}, \vec{x}_{2}, A_{2}, B_{2}\right)=\tilde{G}\left(x_{1}^{4},-\vec{x}_{1}, B_{1}, A_{1} ; x_{2}^{4},-\vec{x}_{2}, B_{2}, A_{2}\right) \text {. }
$$

Let us proceed now to find how these symmetries constraint the form of the propagator. Because of translational invariance $\tilde{G}$ depends on the difference $x_{1}-x_{2}$. The vector $x_{1}-x_{2}$ can be split into a radial and an angular part as follows

$$
x_{1}-x_{2} \rightarrow\left\{\begin{array}{l}
\left|x_{1}-x_{2}\right| \\
X_{12}=\frac{\left(x_{1}^{4}-x_{2}^{4}\right)}{\left|x_{1}-x_{2}\right|}+\mathrm{i} \frac{\left(\mathrm{x}_{1}^{\mathrm{a}}-\mathrm{x}_{2}^{\mathrm{a}}\right)}{\left|\mathrm{x}_{1}-\mathrm{x}_{2}\right|} \tau_{\mathrm{a}} \in \mathrm{SU}(2) .
\end{array}\right.
$$

$\tilde{G}$ is a smooth function of $X_{12}, A_{1}, B_{1}, A_{2}, B_{2}$. Any such function can be decomposed as a linear combination of the matrix elements $D_{\sigma \sigma^{\prime}}^{j}$. This can be seen from group theory or because the $D^{j}$ matrices are the spherical harmonics on $S^{3}$. Thus, we expand $\tilde{G}$ as

$$
\begin{gathered}
\tilde{G}\left(\left|x_{1}-x_{2}\right|, X_{12}, A_{1}, B_{1}, A_{2}, B_{2}\right)=\sum_{j_{i},\left|\sigma_{i}\right|,\left|\sigma_{i}^{\prime}\right| \leq j_{i}} \eta\left(j_{i}, \sigma_{i}, \sigma_{i}^{\prime} ;\left|x_{2}-x_{1}\right|\right) \\
D_{\sigma_{1} \sigma_{1}^{\prime}}^{j_{1}}\left(A_{1}\right) D_{\sigma_{2} \sigma_{2}^{\prime}}^{j_{2}}\left(B_{1}\right) D_{\sigma_{3} \sigma_{3}^{\prime}}^{j_{3}}\left(A_{2}\right) D_{\sigma_{4} \sigma_{4}^{\prime}}^{j_{4}}\left(B_{2}\right) D_{\sigma_{5} \sigma_{5}^{\prime}}^{j_{5}}\left(X_{12}\right)
\end{gathered}
$$

where $j$ runs over $\frac{1}{2} Z_{\geq 0}$, i.e. integer and half-integer representations. From definition (21) we see that

$$
\begin{aligned}
& \tilde{G}\left(x_{1}, A_{1}, B_{1} ; x_{2}, A_{2}, B_{2}\right)=\tilde{G}\left(x_{1},-A_{1},-B_{1} ; x_{2},-A_{2},-B_{2}\right) \\
& \Rightarrow(-)^{2\left(j_{1}+j_{2}+j_{3}+j_{4}\right)}=1,
\end{aligned}
$$

where the implication follows from the formula $D^{j}(-A)=(-)^{2 j} D^{j}(A)$. 
Performing independent variations of $A, S_{1}, S_{2}$ in eqs. (28), (32) and using standard arguments about the coupling of $\mathrm{SU}(2)$ representations one finds that the $\eta$ indices $\left(\sigma_{1} \sigma_{2} \sigma_{3} \sigma_{4}\right)$ are coupled to zero (isorotations), as well as the indices $\left(\sigma_{2}^{\prime}, \sigma_{3}^{\prime}, \sigma_{5}^{\prime}\right)$ and $\left(\sigma_{1}^{\prime}, \sigma_{4}^{\prime},-\sigma_{5}\right)$ (Lorentz transformations). Thus, the most general $\tilde{G}$ satisfying all these symmetries is

$$
\begin{aligned}
& \tilde{G}\left(\left|x_{1}-x_{2}\right|, X_{12}, A_{1}, B_{1}, A_{2}, B_{2}\right)= \\
& \quad \sum_{\sigma_{1}^{\prime}, j, m, I}(2 I+1)^{2} C_{\sigma_{1}^{\prime} \bar{\sigma}_{1}} C_{\bar{\sigma}_{4} \sigma_{4}^{\prime}} C_{m_{2} m_{1}} \eta_{j_{1} j_{2} j_{3} j_{4} j_{5} I}\left(\left|x_{2}-x_{1}\right|\right) \\
& \quad\left(\begin{array}{lll}
j_{1} & j_{4} & j_{5} \\
\bar{\sigma}_{1} & \bar{\sigma}_{4} & \sigma_{5}
\end{array}\right)\left(\begin{array}{ccc}
j_{3} & j_{2} & j_{5} \\
\sigma_{3}^{\prime} & \sigma_{2}^{\prime} & \sigma_{5}^{\prime}
\end{array}\right)\left(\begin{array}{ccc}
j_{1} & j_{2} & I \\
\sigma_{1} & \sigma_{2} & m_{1}
\end{array}\right)\left(\begin{array}{lll}
j_{3} & j_{4} & I \\
\sigma_{3} & \sigma_{4} & m_{2}
\end{array}\right) \\
& D_{\sigma_{1} \sigma_{1}^{\prime}}^{j_{1}}\left(A_{1}\right) D_{\sigma_{2} \sigma_{2}^{\prime}}^{j_{2}}\left(B_{1}\right) D_{\sigma_{3} \sigma_{3}^{\prime}}^{j_{3}}\left(A_{2}\right) D_{\sigma_{4} \sigma_{4}^{\prime}}^{j_{4}}\left(B_{2}\right) D_{\sigma_{5} \sigma_{5}^{\prime}}^{j_{5}}\left(X_{12}\right)
\end{aligned}
$$

where use was made of the Wigner's $3 j$ symbols (which couple to zero three angular momenta) and of the matrices $C_{m m^{\prime}}=(-)^{j+m} \delta_{m,-m^{\prime}}$. Useful properties of $C$ are $C=C^{*}, C^{-1}=C^{t}=(-)^{2 j} C$.

Parity conservation implies $\eta_{j_{1} j_{2} j_{3} j_{4} j_{5} I}=\eta_{j_{2} j_{1} j_{4} j_{3} j_{5} I}$ (cf. eq. (34)).

The transformation laws of this section can also be defined over the fields $\psi_{A, B}, \bar{\psi}_{A, B}$ by means of unitary operators $\mathrm{U}$ satisfying:

Isorotations:

$$
\begin{aligned}
& \mathrm{U}_{\mathrm{A}} \psi_{\mathrm{A}_{1}, \mathrm{~B}_{1}}(\mathrm{x}) \mathrm{U}_{\mathrm{A}}^{\dagger}=\psi_{A A_{1}, A B_{1}}(x), \\
& \mathrm{U}_{\mathrm{A}} \bar{\psi}_{\mathrm{A}_{1}, \mathrm{~B}_{1}}(\mathrm{x}) \mathrm{U}_{\mathrm{A}}^{\dagger}=\bar{\psi}_{A A_{1}, A B_{1}}(x) .
\end{aligned}
$$

Lorentz transformations:

$$
\begin{aligned}
\mathrm{U}_{\Lambda} \psi_{\mathrm{A}_{1}, \mathrm{~B}_{1}}(\mathrm{x}) \mathrm{U}_{\Lambda}^{\dagger} & =\psi_{\mathrm{A}_{1} \mathrm{~S}_{1}^{\dagger}, \mathrm{B}_{1} \mathrm{~S}_{2}^{\dagger}}(\Lambda \mathrm{x}), \\
\mathrm{U}_{\Lambda} \bar{\psi}_{\mathrm{A}_{2}, \mathrm{~B}_{2}}(\mathrm{x}) \mathrm{U}_{\Lambda}^{\dagger} & =\psi_{\mathrm{A}_{1} \mathrm{~S}_{2}^{\dagger}, \mathrm{B}_{1} \mathrm{~S}_{1}^{\dagger}}(\Lambda \mathrm{x}),
\end{aligned}
$$

where $\Lambda \equiv\left(S_{1}, S_{2}\right)$.

These transformations suggest changing to a basis of fields which transform under a finite dimensional representation of the Lorentz and isospin groups. Finite dimensional irreducible representations of the $\mathrm{SU}(2) \times \mathrm{SU}(2)$ group are labeled by two numbers $j_{1}, j_{2} \in \frac{1}{2} Z_{\geq 0}$. If $\Lambda \equiv\left(S_{1}, S_{2}\right)$ the representation $(j, 0)$ is given by $\mathcal{D}^{j}(\Lambda)=D^{j}\left(S_{1}\right)$ and $(0, j)$ by $\overline{\mathcal{D}}^{j}(\Lambda)=D^{j}\left(S_{2}\right)$. The matrices $D^{j}$ are the usual rotation matrices representing $\mathrm{SU}(2)$ and so, unlike the Minkowski case, this representations are unitary but not-faithful. Finally the $\left(j_{1}, j_{2}\right)$ representations are given by $\left(j_{1}, 0\right) \otimes\left(0, j_{2}\right)$. The fields transforming 
under these representations are given by:

$$
\begin{aligned}
\psi_{\sigma_{1} \sigma_{2} m_{I}}^{j_{1} j_{2} I}(x)= & Z_{j_{1} j_{2} I}^{-1} C_{\sigma_{1}^{\prime} \sigma_{1}} C_{\sigma_{2}^{\prime} \sigma_{2}}\left(\begin{array}{ccc}
j_{1} & j_{2} & I \\
\sigma_{1}^{\prime \prime} & \sigma_{2}^{\prime \prime} & m_{I}
\end{array}\right) \\
& \int_{A, B} D_{\sigma_{1}^{\prime \prime} \sigma_{1}^{\prime}}^{j_{1}}(A) D_{\sigma_{2}^{\prime \prime} \sigma_{2}^{\prime}}^{j_{2}}(B) \psi_{A, B}(x),
\end{aligned}
$$

and the same formula for $\bar{\psi}$. The integration over $\mathrm{SU}(2)$ is performed using the group invariant measure and $Z_{j_{1} j_{2} I}$ is a normalization factor to be chosen later. The transformations (39), (40) now read:

$$
\begin{aligned}
\mathrm{U}_{\mathrm{A}} \psi_{\sigma_{1} \sigma_{2} \mathrm{~m}_{\mathrm{I}}}^{\mathrm{j}_{1} \mathrm{j}_{2} \mathrm{I}}(\mathrm{x}) \mathrm{U}_{\mathrm{A}}^{\dagger} & =D_{m_{I}^{\prime} m_{I}}^{I}(A) \psi_{\sigma_{1} \sigma_{2} m_{I}^{\prime}}^{j_{1} j_{2} I}(x), \\
\mathrm{U}_{\mathrm{A}} \bar{\psi}_{\sigma_{1} \sigma_{2} \mathrm{~m}_{\mathrm{I}}}^{\mathrm{j}_{1} \mathrm{j}_{2} \mathrm{I}}(\mathrm{x}) \mathrm{U}_{\mathrm{A}}^{\dagger} & =D_{m_{I}^{\prime} m_{I}}^{I}(A) \psi_{\sigma_{1} \sigma_{2} m_{I}^{\prime}}^{j_{1} j_{2} I}(x), \\
\mathrm{U}_{\Lambda} \psi_{\sigma_{1} \sigma_{2} \mathrm{~m}_{\mathrm{I}}}^{\mathrm{j}_{1} \mathrm{j}_{2} \mathrm{I}}(\mathrm{x}) \mathrm{U}_{\Lambda}^{\dagger} & =\mathcal{D}_{\sigma_{1} \sigma_{1}^{\prime}}^{j_{1}}\left(\Lambda^{-1}\right) \overline{\mathcal{D}}_{\sigma_{2} \sigma_{2}^{\prime}}^{j_{2}}\left(\Lambda^{-1}\right) \psi_{\sigma_{1}^{\prime} \sigma_{2}^{\prime} m_{I}}^{j_{1} j_{2} I}(\Lambda x), \\
\mathrm{U}_{\Lambda} \bar{\psi}_{\sigma_{1} \sigma_{2} \mathrm{~m}_{\mathrm{I}}}^{\mathrm{j}_{1} \mathrm{j}_{2} \mathrm{I}}(\mathrm{x}) \mathrm{U}_{\Lambda}^{\dagger} & =\overline{\mathcal{D}}_{\sigma_{1} \sigma_{1}^{\prime}}^{j_{1}}\left(\Lambda^{-1}\right) \mathcal{D}_{\sigma_{2} \sigma_{2}^{\prime}}^{j_{2}}\left(\Lambda^{-1}\right) \bar{\psi}_{\sigma_{1}^{\prime} \sigma_{2}^{\prime} m_{I}}^{j_{1} j_{2} I}(\Lambda x),
\end{aligned}
$$

which means that $\psi_{\sigma_{1} \sigma_{2} m_{I}}^{j_{1} j_{2} I}(x)$ transforms under the Lorentz group representation $\left(j_{1}, j_{2}\right)$ and $\bar{\psi}_{\sigma_{1} \sigma_{2} m_{I}}^{j_{1} j_{2} I}(x)$ under the representation $\left(j_{2}, j_{1}\right)$. In addition, both fields have isospin $I$.

Using eq. (38) and definition (41) we can obtain the general form of the propagator

$$
\begin{aligned}
\left\langle 0\left|T\left\{\bar{\psi}_{\sigma_{3} \sigma_{4} m_{I}^{\prime}}^{j_{3} j_{4} I^{\prime}} \psi_{\sigma_{1} \sigma_{2} m_{I}}^{j_{1} j_{2} I}\right\}\right| 0\right\rangle= & Z_{j_{1} j_{2} I}^{-1} Z_{j_{3} j_{4} I^{\prime}}^{-1} \delta_{I I^{\prime}} C_{m_{I}^{\prime} m_{I}} D_{\sigma_{1} \sigma_{1}^{\prime}}^{j_{1}}\left(X_{12}\right) D_{\sigma_{4} \sigma_{4}^{\prime}}^{j_{4}}\left(X_{12}\right) \\
& \sum_{j_{6}}\left(\begin{array}{ccc}
j_{1} & j_{2} & j_{6} \\
\sigma_{1}^{\prime} & \sigma_{2} & \sigma_{6}
\end{array}\right)\left(\begin{array}{lll}
j_{3} & j_{4} & j_{6} \\
\sigma_{3} & \sigma_{4}^{\prime} & \sigma_{6}^{\prime}
\end{array}\right) C_{\sigma_{6}^{\prime} \sigma_{6}} \bar{\eta}_{j_{1} j_{2} j_{3} j_{4} j_{6} I}
\end{aligned}
$$

where $\bar{\eta}$ can be written in terms of $\eta$ and the $6 j$ symbols [15] as

$$
\bar{\eta}_{j_{1} j_{2} j_{3} j_{4} j_{6} I}=\sum_{j_{5}} \eta_{j_{1} j_{2} j_{3} j_{4} j_{5} I}(-)^{2 j_{5}}\left\{\begin{array}{ccc}
j_{1} & j_{2} & j_{6} \\
j_{3} & j_{4} & j_{5}
\end{array}\right\} \text {. }
$$

The spins associated with these fields are obtained by composing $j_{1}$ and $j_{2}$ and so, in principle, they range from $\left|j_{2}-j_{1}\right|$ to $j_{1}+j_{2}$. The propagated spins are determined by the propagator in (16). In the next section we will show that (16) has a new symmetry which leads to the propagation of particles with spin equal to the isospin $I$. 


\section{Skyrmion propagator}

Now, we will approximately evaluate the propagator in the large $\left|x_{2}-x_{1}\right|$ limit. Performing a Lorentz transformation, the 4-axis can always be taken along $x_{2}-x_{1}$, i.e. $X_{12}=1$. By means of eq. (43) we can extend this result to arbitrary values of $X_{12}$.

Having fixed $X_{12}$, we will analize what is expected for different values of $A_{1,2}$ and $B_{1,2}$.

If $A_{1}=B_{1}=A_{2}=B_{2}=1$, the situation is that of section 2 where we evaluated (numerically) the saddle point. This saddle point will be the base for our analysis and will be denoted as $U_{0}(t, \vec{x})$. One should remember that it behaves as $-X_{2}^{\dagger}$ around $x_{2}$ and as $X_{1}$ around $x_{1}$. Note also that $U_{0}(t, \vec{x})$ (as a function of $\vec{x}$ ), in the time interval between $x_{2}^{4}$ and $x_{1}^{4}$, is almost equal to the static soliton profile, which will be denoted as $U_{\mathrm{SK}}(\vec{x})$.

Suppose now that $A_{2}=B_{2}=A, A_{1}=B_{1}=1$. This corresponds to performing an isorotation in the vicinity of $x_{2}$. We know that if $U$ is a solution to the equation of motion, then, $A U A^{\dagger}$ (with constant $A$ ) is also a solution, that is, the function $A U_{0} A^{\dagger}$ satisfies the equations of motion around $x_{2}$. It also satisfies the boundary condition on $\partial B_{2}^{4}$. Moreover, this behavior can be matched with the behavior around $x_{1}$ by means of a time dependent ansatz of the form

$$
U(t, \vec{x})=A(t) U_{0} A^{\dagger}(t), \quad A\left(x_{2}^{4}\right)=A, A\left(x_{1}^{4}\right)=1,
$$

which satisfies $U=1$ at infinity; upon substitution in the action we obtain an equation for $A(t)$. The corresponding solution will represent, through eq. (45), the creation at $x_{2}$ of a skyrmion isorotated in $A$, with profile $A U_{\mathrm{SK}} A^{\dagger}$, which slowly rotates until it is annihilated unrotated at $x_{1}$. This solution is expected to give a good approximation to the saddle point. More generally, for $A_{2}=B_{2}, A_{1}=B_{1}$ the saddle point will be approximately given by minimizing the action with respect to the ansatz (45) with $A\left(x_{2}^{4}\right)=A_{2}, A\left(x_{1}^{4}\right)=A_{1}$. This is by now a standard calculation in skyrmion physics which gives free motion over $\mathrm{SU}(2)$ with a moment of inertia $\Im$, obtained from $U_{\mathrm{SK}}(\vec{x})$ [1]. A better approximation to the field propagator can be done by integrating over all possible $A(t)$ giving the free propagator over $\mathrm{SU}(2)$, from $A_{2}$ to $A_{1}$, which corresponds to quantizing the isorotational (or rotational) zero-modes.

Now, we consider the case $A_{2} \neq B_{2}, A_{1}=B_{1}=1$. In this case we also have that $A_{2} U_{0} B_{2}^{\dagger}$ is a solution in the vicinity of $x_{2}$ that satisfies the correct boundary condition at $\partial B_{2}^{4}$ but here, a time dependent ansatz does not suffices; for such an ansatz, the behavior $A_{2} U_{0} B_{2}^{\dagger}$ around the vicinity of $x_{2}$ would be extended to infinity on the hyperplanes $x^{4}=$ const. $\approx \mathrm{x}_{2}^{4}$ to a value $A_{2} B_{2}^{\dagger} \neq 1$ that does not match the required asymptotic behavior of the fields. Recall that, around 
$x_{2}, U_{0}$ is unlocalized for $\left|x-x_{2}\right|<r_{l}$, while it is localized on the North pole for $\left|x-x_{2}\right|>r_{l}$ (see the discussion at the end of section 2). Then, if we start with a function such that, very close to $\left|x-x_{2}\right|=\epsilon$, behaves as the solution $A_{2} U_{0} B_{2}^{\dagger}$ and this behavior persists up to the localization radius, the topological density will not be localized on the North pole of the sphere $\left|x-x_{2}\right|=r_{l}$, but on the direction $x-x_{2}$ satisfying $X_{2}=B_{2}^{\dagger} A_{2}$. This behavior represents a soliton that starts moving on a direction other than towards $x_{1}$. Therefore, the matching with the behavior around $x_{1}$ where the soliton is required to be annihilated will lead to a function with action larger than the action associated with a function representing a soliton that starts moving towards $x_{1}$, that is, we expect that the configuration which minimizes the action behaves, in the unlocalization region around $x_{2}$, as

$$
U(x)=A(\tau) U_{0} B(\tau)^{\dagger}, \quad A(\epsilon)=A_{2}, B(\epsilon)=B_{2}, \quad A\left(r_{l}\right)=B\left(r_{l}\right)=C_{2}
$$

where $\tau=\left|x-x_{2}\right|, \epsilon$ is the radius of the small sphere we extracted around $x_{2}$ and $r_{l} \sim\left(e f_{\pi}\right)^{-1}$. Note that this behavior, at $\tau=r_{l}$, correctly localizes the topological density on the North pole, though isorotated by $C_{2}$. The value of $C_{2}$ must be chosen so as to minimize the generated action in the unlocalization region. Loosely speaking, $\left(C_{2}, C_{2}\right)$ will be the closest rotation to the Lorentz transformation $\left(A_{2}, B_{2}\right)$. A more quantitative answer is obtained by inserting the above ansatz in the action, leading to

$$
\begin{aligned}
S & =4 \pi^{2} \int_{\epsilon<\tau<r_{l}} \mathrm{~d} \tau \mathrm{h}(\tau) \operatorname{Tr}\left(\dot{\mathrm{A}} \dot{\mathrm{A}}^{\dagger}+\dot{\mathrm{B}}^{\dagger}\right), \\
h(\tau) & =\frac{f_{\pi}^{2}}{8} \tau^{3}+\frac{\tau}{4 e^{2}}-\frac{\epsilon_{6}}{16 \pi^{2}} \frac{1}{\tau},
\end{aligned}
$$

where the dots represent derivation with respect to $\tau$, and we made the approximation $U_{0} \simeq-X_{2}^{\dagger}$. After a change of variables $u=u(\tau)$ such that $\dot{u}=1 / h(\tau)$, which cancels the factor $h(\tau)$, the expression (47) represents free motion over $\mathrm{SU}(2) \times \mathrm{SU}(2)$ for $(A(u), B(u))$. For small $\tau$, the solution to $\dot{u}=1 / h(\tau)$ is $u(\tau) \simeq-8 \pi^{2} \tau^{2} / \epsilon_{6}$.

At $u \sim u\left(r_{l}\right)$ we must have $A(u)=B(u)=C_{2}$, then the action is equal to

$$
\begin{aligned}
S & =8 \pi^{2} \frac{s_{A_{2} C_{2}}^{2}+s_{B_{2} C_{2}}^{2}}{u\left(r_{l}\right)-u(\epsilon)}, \\
s_{A B} & =2 \operatorname{Arccos}\left(\frac{1}{2} \operatorname{Tr}\left(\mathrm{AB}^{\dagger}\right)\right) .
\end{aligned}
$$

Where $s_{A B}$ is the distance between $A$ and $B$ measured with the standard metric of $\mathrm{SU}(2)$. For $A_{2}, B_{2}$ fixed, a calculation reveals that this action is minimized by $C_{2}=B_{2} \sqrt{B_{2} A_{2}^{\dagger}}$. Note that in $\mathrm{SU}(2)$, the square root is well defined up to a signf. This part of the saddle point action contributes to the

${ }^{4}$ except for the square root of minus the identity since any matrix $A=i \vec{a} \cdot \vec{\tau}, \vec{a} \cdot \vec{a}=1$, 
propagator with a factor $\exp \left(-\left(s_{A_{2} C_{2}}^{2}+s_{B_{2} C_{2}}^{2}\right) /\left(u\left(r_{l}\right)-u(\epsilon)\right)\right.$. In a general situation, where $A_{1} \neq B_{1}$, the same analysis is also valid around $x_{1}$. The results are best expressed in terms of the minimizing variables $C_{i}=B_{i} \sqrt{B_{i}^{\dagger} A_{i}}$ and the variables $D_{i}$, defined by the relations

$$
A_{i}=C_{i} D_{i}, \quad B_{i}=C_{i} D_{i}^{\dagger}
$$

which yields $D_{i}=\sqrt{B_{i}^{\dagger} A_{i}}$. For the minimizing $C_{i}$ 's we have

$$
s_{A_{i} C_{i}}^{2}+s_{B_{i} C_{i}}^{2}=\frac{s_{A_{i} B_{i}}^{2}}{2}=2 \operatorname{Arccos}\left(\frac{1}{2} \operatorname{Tr}\left(\mathrm{D}_{\mathrm{i}}^{2}\right)\right)
$$

Now, the behaviors (at $r_{l}$ ) around $x_{1}$ and $x_{2}$ can be matched by means of a time dependent ansatz whose minimization will complete the construction of the saddle point and will represent a slowly rotating soliton from $C_{2}$ to $C_{1}$. Again, a better approximation is obtained by considering free propagation over $\mathrm{SU}(2)$ from $C_{2}$ to $C_{1}$ (whose expression can be found in [16]); including the factors generated in the unlocalization region, we obtain the following expression for $\tilde{G}$

$$
\begin{aligned}
\tilde{G}\left(x_{1}, A_{1}, B_{1} ; x_{2}, A_{2}, B_{2}\right)= & g_{1}\left(\operatorname{TrD}_{1}\right) \mathrm{g}_{2}\left(\operatorname{TrD}_{2}\right) \sum_{\overline{\overline{\mathrm{I}}}} \mathrm{D}_{\mathrm{km}}^{\overline{\mathrm{I}}}\left(\mathrm{C}_{1}\right) \mathrm{D}_{\mathrm{mk}}^{\overline{\overline{\mathrm{I}}}}\left(\mathrm{C}_{2}^{\dagger}\right) \\
& \mathrm{e}^{-\frac{\overline{\mathrm{I}}(\overline{\mathrm{I}}+1)}{2 \Im}\left|\mathrm{x}_{2}-\mathrm{x}_{1}\right|} \frac{\mathrm{e}^{-\mathrm{M}_{\mathrm{cl}}\left|\mathrm{x}_{2}-\mathrm{x}_{1}\right|}}{\left(\frac{2 \pi}{\mathrm{M}}\left|\mathrm{x}_{2}-\mathrm{x}_{1}\right|\right)^{3 / 2}}
\end{aligned}
$$

where we also include the factor $\exp -M_{\mathrm{cl}}\left|x_{2}-x_{1}\right|$ coming from the classical action, and the factor proportional to $\left|x_{2}-x_{1}\right|^{-3 / 2}$ resulting from the integration over the translational zero-modes. The function $g_{i}$ only depends on $\operatorname{Tr}\left(\mathrm{D}_{\mathrm{i}}\right)$, since for an $\mathrm{SU}(2)$ matrix $\operatorname{Tr}\left(\mathrm{D}^{2}\right)=(\operatorname{Tr}(\mathrm{D}))^{2}-2$, and contains the gaussian factor $\exp \left(-\left(s_{A_{i} C_{i}}^{2}+s_{B_{i} C_{i}}^{2}\right) /\left(u\left(r_{l}\right)-u(\epsilon)\right)\right.$ (cf. eq. (50)).

Finally, to obtain the propagator $G$, we note that $A \rightarrow-A, B \rightarrow-B$ is equivalent to $C \rightarrow-C, D \rightarrow D$, and $D^{\bar{I}}(-C)=(-)^{2 \bar{I}} D^{\bar{I}}(C)$; therefore, if we define $G$ in eq. (22) using the plus (minus) sign the only surviving terms are those with integer (half-integer) $\bar{I}$. We will show below that $\bar{I}$ is the spin and isospin, thus choosing the plus (minus) sign will lead to integer (half-integer) spin (equal to isospin) particles.

Note that $\tilde{G}$ in equation (51) enjoys the two following properties: (1) $\tilde{G}$ satisfies the parity requirement (34) (invariance under $A_{i} \leftrightarrow B_{i}$ ) since this is equivalent to $D_{i} \leftrightarrow D_{i}^{\dagger}$ and for an $\mathrm{SU}(2)$ matrix $\operatorname{Tr}(\mathrm{D})=\operatorname{Tr}\left(\mathrm{D}^{\dagger}\right),(2) \tilde{G}$ is invariant under the new symmetry $D_{i} \rightarrow R_{i} D_{i} R_{i}^{\dagger}$.

satisfies $A^{2}=-1$. 
Now we will show that the propagator of eq. (51) propagates spin equal to isospin particles.

To find the propagator of the fields $\psi^{j_{1} j_{2}}$ we use eq. (41) to obtain:

$$
\begin{gathered}
\left\langle 0\left|T\left\{\bar{\psi}_{\sigma_{3} \sigma_{4} m_{I}^{\prime}}^{j_{3} j_{4} I^{\prime}} \psi_{\sigma_{1} \sigma_{2} m_{I}}^{j_{1} j_{2} I}\right\}\right| 0\right\rangle=Z_{j_{1} j_{2} I}^{-1} Z_{j_{3} j_{4} I^{\prime}}^{-1} \sum_{\bar{I}}\left(\begin{array}{ccc}
j_{3} & j_{4} & I^{\prime} \\
\sigma_{3}^{\prime \prime} & \sigma_{4}^{\prime \prime} & m_{I}^{\prime}
\end{array}\right)\left(\begin{array}{ccc}
j_{1} & j_{2} & I \\
\sigma_{1}^{\prime \prime} & \sigma_{2}^{\prime \prime} & m_{I}
\end{array}\right) \\
C_{\sigma_{1}^{\prime} \sigma_{1}} C_{\sigma_{2}^{\prime} \sigma_{2}} C_{\sigma_{3}^{\prime} \sigma_{3}} C_{\sigma_{4}^{\prime} \sigma_{4}} \int_{A_{1,2} B_{1,2}} D_{\sigma_{1}^{\prime \prime} \sigma_{1}^{\prime}}^{j_{1}}\left(A_{1}\right) D_{\sigma_{2}^{\prime \prime} \sigma_{2}^{\prime}}^{j_{2}}\left(B_{1}\right) D_{\sigma_{3}^{\prime \prime} \sigma_{3}^{\prime}}^{j_{3}}\left(A_{2}\right) D_{\sigma_{4}^{\prime \prime} \sigma_{4}^{\prime}}^{j_{4}}\left(B_{2}\right) \\
g_{1}\left(\operatorname{Tr}_{1}\right) \mathrm{g}_{2}\left(\operatorname{TrD}_{2}\right) \mathrm{D}_{\mathrm{km}}^{\overline{\mathrm{I}}}\left(\mathrm{C}_{1}\right) \mathrm{D}_{\mathrm{mk}}^{\overline{\mathrm{I}}}\left(\mathrm{C}_{2}^{\dagger}\right) \frac{\mathrm{e}^{-\mathrm{M}_{\overline{\mathrm{I}}\left|\mathrm{x}_{2}-\mathrm{x}_{1}\right|}}}{\left(\frac{2 \pi}{\mathrm{M}}\left|\mathrm{x}_{2}-\mathrm{x}_{1}\right|\right)^{3 / 2}},
\end{gathered}
$$

where we introduced $M_{\bar{I}}=M_{\mathrm{cl}}+\bar{I}(\bar{I}+1) /(2 \Im)$. Let us first perform the integral over $A_{1}, B_{1}$. It is convenient to write everything in terms of $C_{1}$ and $D_{1}$, the factors involved are:

$$
\begin{aligned}
& \left(\begin{array}{lll}
j_{1} & j_{2} & I \\
\sigma_{1}^{\prime \prime} & \sigma_{2}^{\prime \prime} & m_{I}
\end{array}\right) C_{\sigma_{1}^{\prime} \sigma_{1}} C_{\sigma_{2}^{\prime} \sigma_{2}} \times \\
& \times \int_{A_{1} B_{1}} D_{\sigma_{1}^{\prime \prime} \sigma_{1}^{\prime}}^{j_{1}}\left(C_{1} D_{1}\right) D_{\sigma_{2}^{\prime \prime} \sigma_{2}^{\prime}}^{j_{2}}\left(C_{1} D_{1}^{\dagger}\right) g_{1}\left(\operatorname{TrD}_{1}\right) \mathrm{D}_{\mathrm{km}}^{\overline{\mathrm{I}}}\left(\mathrm{C}_{1}\right) .
\end{aligned}
$$

To change variables we must evaluate a jacobian which can be calculated parametrizing the matrices as $A=a_{0}+\mathrm{i} \overrightarrow{\mathrm{a}} \cdot \vec{\tau}$ with $\left(a_{0}^{2}+\vec{a}^{2}=1\right)$ and similar expressions for $B, C$ and $D$. The invariant measure is written as

$$
\mathrm{dA}=\frac{\mathrm{da}_{1} \mathrm{da}_{2} \mathrm{da}_{3}}{\mathrm{a}_{0}}
$$

The jacobian is the determinant of a $6 \times 6$ matrix which upon evaluation gives:

$$
\int \mathrm{dAdB}=\int \mathrm{dC} \mathrm{dD} 2(\operatorname{TrD})^{2}
$$

We absorb this factor in $g_{1}$ defining $\bar{g}_{1}=2 g_{1}\left(\operatorname{TrD}_{1}\right)^{2}$. Since $\bar{g}_{1}$ is a class function of $D_{1}$, that is $\bar{g}_{1}\left(S D_{1} S^{\dagger}\right)=\bar{g}_{1}\left(D_{1}\right)$, it can be expanded according to

$$
\bar{g}_{1}\left(D_{1}\right)=\sum_{l_{1}} \bar{g}_{1 l_{1}} D_{\bar{m} \bar{m}}^{l_{1}}\left(D_{1}\right)
$$

We are now ready to evaluate the integrals since the integral of the product of three $D$ matrices is given by the $3 j$ symbols [15]. The result is

$$
\sum_{l_{1}} \bar{g}_{1 l_{1}} C_{\sigma_{1}^{\prime} \sigma_{1}} C_{\sigma_{2}^{\prime \prime \prime} \sigma_{2}^{\prime}}
$$




$$
\left(\begin{array}{lll}
j_{1} & j_{2} & I \\
\sigma_{1}^{\prime \prime} & \sigma_{2}^{\prime \prime} & m_{I}
\end{array}\right)\left(\begin{array}{lll}
j_{1} & j_{2} & \bar{I} \\
\sigma_{1}^{\prime \prime} & \sigma_{2}^{\prime \prime} & k
\end{array}\right)\left(\begin{array}{ccc}
j_{1} & j_{2} & \bar{I} \\
\sigma_{1}^{\prime \prime \prime} & \sigma_{2}^{\prime \prime \prime} & m
\end{array}\right)\left(\begin{array}{lll}
j_{1} & j_{2} & l_{1} \\
\sigma_{1}^{\prime \prime \prime} & \sigma_{2} & \bar{m}
\end{array}\right)\left(\begin{array}{lll}
j_{1} & j_{2} & l_{1} \\
\sigma_{1}^{\prime} & \sigma_{2}^{\prime} & \bar{m}
\end{array}\right)
$$

The two first $3 j$ symbols give $\delta_{I \bar{I}} \delta_{m_{I} k} /(2 I+1)$, the three remaining symbols can be simplified by means of the $6 j$ symbol to give

$$
\sum_{l_{1}} \frac{\bar{g}_{1 l_{1}}}{2 I+1} C_{\sigma_{1} \sigma_{1}^{\prime}} C_{\sigma_{2} \sigma_{2}^{\prime}} \delta_{k m_{I}}\left\{\begin{array}{ccc}
j_{1} & j_{2} & I \\
j_{1} & j_{2} & l_{1}
\end{array}\right\}\left(\begin{array}{lll}
j_{1} & j_{2} & I \\
\sigma_{1}^{\prime} & \sigma_{2}^{\prime} & m
\end{array}\right)
$$

The integral over $A_{2}, B_{2}$ can be performed in a similar way. Putting all the pieces together we obtain:

$$
\begin{gathered}
\left\langle 0\left|T\left\{\bar{\psi}_{\sigma_{3} \sigma_{4} m_{I}^{\prime}}^{j_{3} j_{4} I^{\prime}} \psi_{\sigma_{1} \sigma_{2} m_{I}}^{j_{1} j_{j} I}\right\}\right| 0\right\rangle=Z_{j_{1} j_{2} I}^{-1} \bar{Z}_{j_{3} j_{4} I^{\prime}}^{-1} \frac{C_{m_{I}^{\prime} m_{I}} \delta_{I I^{\prime}}}{(2 I+1)^{2}} \frac{\mathrm{e}^{-\mathrm{M}_{\mathrm{I}}\left|\mathrm{x}_{2}-\mathrm{x}_{1}\right|}}{\left(\frac{2 \pi}{M}\left|x_{2}-x_{1}\right|\right)^{3 / 2}} \\
\left(\begin{array}{lll}
j_{1} & j_{2} & I \\
\sigma_{1} & \sigma_{2} & m
\end{array}\right)\left(\begin{array}{ccc}
j_{3} & j_{4} & I \\
\sigma_{3} & \sigma_{4} & m^{\prime}
\end{array}\right) C_{m^{\prime} m} \sum_{l_{1} l_{2}} \bar{g}_{1 l_{1}} \bar{g}_{2 l_{2}}\left\{\begin{array}{lll}
j_{1} & j_{2} & I \\
j_{1} & j_{2} & l_{1}
\end{array}\right\}\left\{\begin{array}{lll}
j_{3} & j_{4} & I \\
j_{3} & j_{4} & l_{2}
\end{array}\right\} .
\end{gathered}
$$

Choosing in this equation

$$
Z_{j_{1} j_{2} I}=\sum_{l_{1}} \frac{\bar{g}_{1 l_{1}}}{2 I+1}\left\{\begin{array}{lll}
j_{1} & j_{2} & I \\
j_{1} & j_{2} & l_{1}
\end{array}\right\}
$$

and the same for $Z_{j_{3} j_{4} I^{\prime}}$ (replacing $\bar{g}_{1 l_{1}}$ by $\bar{g}_{2 l_{2}}$ ) we arrive at

$$
\left(\begin{array}{lll}
j_{1} & j_{2} & I \\
\sigma_{1} & \sigma_{2} & m
\end{array}\right)\left(\begin{array}{lll}
j_{3} & j_{4} & I \\
\sigma_{3} & \sigma_{4} & m^{\prime}
\end{array}\right) C_{m^{\prime} m} C_{m_{I}^{\prime} m_{I}} \delta_{I I^{\prime}} \frac{\mathrm{e}^{-\mathrm{M}_{\mathrm{I}}\left|\mathrm{x}_{2}-\mathrm{x}_{1}\right|}}{\left(\frac{2 \pi}{M}\left|x_{2}-x_{1}\right|\right)^{3 / 2}} .
$$

Finally, from eq. (43) we can read the value of $\bar{\eta}$ and then extend the propagator from $X_{12}=1$ to an arbitrary $X_{12}$, obtaining

$$
\begin{aligned}
\left\langle 0\left|T\left\{\bar{\psi}_{\sigma_{3} \sigma_{4} m_{I}^{\prime}}^{j_{3} j_{4} I^{\prime}} \psi_{\sigma_{1} \sigma_{2} m_{I}}^{j_{1} j_{2} I}\right\}\right| 0\right\rangle= & C_{m^{\prime} m} C_{m_{I}^{\prime} m_{I}} \delta_{I I^{\prime}} \mathcal{D}_{\sigma_{1} \sigma_{1}^{\prime}}^{j_{1}}\left(X_{12}\right) \mathcal{D}_{\sigma_{4} \sigma_{4}^{\prime}}^{j_{4}}\left(X_{12}\right) \\
& \left(\begin{array}{ccc}
j_{1} & j_{2} & I \\
\sigma_{1}^{\prime} & \sigma_{2} & m
\end{array}\right)\left(\begin{array}{lll}
j_{3} & j_{4} & I \\
\sigma_{3} & \sigma_{4}^{\prime} & m^{\prime}
\end{array}\right) \frac{\mathrm{e}^{-\mathrm{M}_{\mathrm{I}}\left|\mathrm{x}_{2}-\mathrm{x}_{1}\right|}}{\left(\frac{2 \pi}{M}\left|x_{2}-x_{1}\right|\right)^{3 / 2}}(62)
\end{aligned}
$$

This expression coincides with the free field propagator we evaluate in appendix A, namely, formula (A.15), where the index $I$ in (62) replaces the index $j$ in that formula. The index $j$ in (A.15) is the spin of the one particle states we use in the appendix to construct the free fields, on the other hand, 
$I$ is the isospin of our fields (cf. eq. (42)), implying that the propagator $\tilde{G}$ describes a tower of spin equal to isospin particles (with mass $M_{I}$ ). Furthermore, the Green functions obtained in the appendix correspond to free fields that for integer (half-integer) $j$ commute (anticommute) at space-like separations, that is, the fields we define by means of the Green functions (23) and eq. (41) obey the spin-statistics theorem. For example, one can calculate from eq. (62) the propagator in the $(1 / 2,0) \oplus(0,1 / 2)$ representation which coincides, at large distances, with the usual Dirac propagator for a spin 1/2 particle.

\section{S-matrix definition}

In order to compute scattering amplitudes one should be able to extract the S-matrix from the Green functions previously defined. As a first step we apply definition (41) to obtain Green functions for the fields $\psi^{j_{1} j_{2}}$ and $\bar{\psi}^{j_{1} j_{2}}$ (remember that $\psi$ and $\bar{\psi}$ refer to singularities of topological number +1 and -1 respectively). Then, the Green functions are analytically continued to Minkowski space-time by the replacement $x^{4} \rightarrow-\mathrm{ix}^{0}$. The large distance propagator, obtained in the previous section, allows us to identify the asymptotic states to be scattered, in the non-trivial topological sector, as composed by spin equal to isospin particles. Now, all we need is the corresponding LSZ reduction formula, which is obtained in the appendix and reads

$$
\begin{aligned}
& { }_{\text {out }}\left\langle p_{1} \bar{\sigma}_{1} \bar{s}_{1}, \cdots, p_{n} \bar{\sigma}_{n} \bar{s}_{n} \mid q_{1} \sigma_{1} s_{1}, \cdots, q_{l} \sigma_{l} s_{l}\right\rangle_{\mathrm{in}}=\text { disc. parts }+\left(\mathrm{iZ}^{-1 / 2}\right)^{\mathrm{n}+\mathrm{l}} \\
& \int \mathrm{d}^{4} \mathrm{y}_{1} \cdots \mathrm{d}^{4} \mathrm{x}_{\mathrm{l}} \overline{\mathrm{u}}_{\mathrm{m}_{1} \mathrm{~m}_{1}^{\prime} \mathrm{j}_{1}^{\prime} \mathrm{I}_{1}}\left(\mathrm{p}_{1}, \bar{\sigma}_{1}\right) \cdots \mathrm{u}_{\mathrm{m}_{l} \mathrm{~m}_{1}^{\prime}}^{\mathrm{j}_{\mathrm{j}} \mathrm{I}_{\mathrm{I}}}\left(\mathrm{q}_{\mathrm{l}}, \sigma_{1}\right) \exp \left(\mathrm{i} \sum_{1}^{\mathrm{n}} \mathrm{p}_{\mathrm{k}} \mathrm{y}_{\mathrm{k}}-\mathrm{i} \sum_{1}^{1} \mathrm{q}_{\mathrm{r}} \mathrm{x}_{\mathrm{r}}\right) \\
& \left(\square_{y_{1}}+m_{1}^{2}\right) \cdots\left(\square_{x_{l}}+m_{l}^{2}\right)\left\langle 0\left|T \bar{\psi}_{m_{1} m_{1}^{\prime} \bar{s}_{1}}^{j_{1} j_{1}^{\prime} I_{1}}\left(y_{1}\right) \cdots \psi_{m_{l} m_{l}^{\prime} s_{l}}^{j_{l} j_{l}^{\prime} I_{l}}\left(x_{l}\right)\right| 0\right\rangle,
\end{aligned}
$$

where $\sigma_{i}$ stands for the spin projection $z$ in the rest frame and $s$ is the isospin $3^{r d}$ projection. The wave functions $u, \bar{u}$ are defined in the appendix; when considering the scattering of antiparticles, $u, \bar{u}$ must be replaced by $v, \bar{v}$. The Green function in eq. (63) is obtained from (24) and (41). An intuitive understanding of this Green function can be achieved using the line defects picture of ref. [8]. There, the leading contribution to the path integral (24) is identified with configurations described by open line defects emerging from $x_{1}, \ldots, x_{n}$ plus a gas of closed defect tubes. If one brings, e.g., $x_{1}$ far from the other points, the sum over the open line defects associated with $x_{1}$ will give a skyrmion free propagator, from $x_{1}$ towards the interaction region. This propagator has the pole (in $p^{2}$ ) required for a non-vanishing scattering amplitude and also contains all information about the boundary condition around $x_{1}$.

As it is noticed in the appendix, the different fields that can be built from

the same particle states are not independent and one should choose only one 
$\psi^{j_{1} j_{2} I}$ to represent each kind of particle. For example the $\Delta(j=3 / 2)$ can be represented by a $(3 / 2,0)$ field or by a $(1,1 / 2)$, as in the Rarita-Schwinger representation. Choosing one or the other amounts to a field redefinition and hence the S-matrix should be independent of this choice. This assertion can be checked, within the saddle point approximation, taking into account that all information about the boundary conditions is contained in the free propagators (62) attached to the points $x_{i}$ (as described in the previous paragraph). The explicit dependence on $\left(j_{1}, j_{2}\right)$ is cancelled by the choice of normalization factor $Z_{j_{1} j_{2} I}$ 60).

It is interesting to note that, for a $(1 / 2,0)$ field, the reduction formula we give contains an operator $\left(\square+m^{2}\right)$, instead of the usual $(\mathrm{i} \not \partial+\mathrm{m})$. This comes about because we are using a two-component spinor, see [17 for a discussion on this point. For a two-component spinor we need $\psi^{1 / 20}$ and $\partial_{t} \psi^{1 / 20}$ to recover the operators $a$ and $b^{\dagger}$. In the case of spin $1 / 2$ fermions, the usual alternative approach is considering two fields, namely $\psi^{1 / 2} 0$ and $\psi^{01 / 2}$ (or in general $\psi^{j_{1} j_{2}}$ and $\psi^{j_{2} j_{1}}$ ). Using these two fields (4-component spinors) one can obtain $a$ and $b^{\dagger}$ with no need of the field time derivative. Then, in that case, the LSZ reduction formula displays a first order operator. The same can be done for higher spin fields but the operator appearing in the LSZ formula will be of higher order and more cumbersome to use than the operator $\left(\square+m^{2}\right)$ associated with a single field representation.

The Green functions we defined in eq. (24) imply that our pion field is not just the fluctuating part but the sum of the classical configuration plus the fluctuating part. In fact, this is the natural thing to do, the separation between classical and fluctuating parts is just a consequence of the saddle point approximation, which may not always be adequate.

Precisely, the suggestion that the pion field be identified with the classical soliton plus fluctuations, instead of identifying it just with the fluctuations, led to the solution to the Yukawa problem (see refs. [0], [18] and [19]).

The Yukawa coupling is obtained from the vertex

$$
\left\langle 0\left|T\left\{\psi_{\sigma_{1}, \sigma_{2}, m_{I}}^{j_{1}, j_{2}, I}\left(x_{1}\right) \bar{\psi}_{\sigma_{4}, \sigma_{3}, m_{I}^{\prime}}^{j_{4}, j_{3}, I^{\prime}}\left(x_{2}\right) \phi_{a}\left(y_{1}\right)\right\}\right| 0\right\rangle,
$$

At large distances $\left(\left|x_{1}-x_{2}\right|\right.$ large $)$ the relevant configurations are described in terms of open line defects which can be interpreted as skyrmion world lines. Then, in this case, the saddle point calculation is similar to that of reference [7] where the correct Yukawa coupling was obtained resorting to a rotationally improved ansatz. In our formalism, the calculation would be anologous to the evaluation of the propagator performed in section 5 .

At short distances $\left(\left|x_{1}-x_{2}\right|\right.$ small $)$ the saddle point no longer resembles a static solution in the interval between creation and destruction of the soliton, 
so one should expect differences with the approach of [7]. However we will not pursue this point here since at short distances the Skyrme lagrangian is not adequate. However, this discussion may be relevant in connection with other solitonic models.

\section{Conclusions}

In ref. [8], Fröhlich and Marchetti proposed a quantum field theory for skyrmions, defining Euclidean Green functions in terms of path integrals over singular fields.

In the present paper we show that by fixing the boundary conditions around the singularities it is possible to study the physical content of the Skyrme model and their dynamical implications in a manifestly covariant way. In particular, we obtain the covariant baryon propagator. This treatment may also be relevant when studying other solitonic models as for example monopoles.

Our method is based on defining Green functions subject to a minimal set of boundary conditions that close under four dimensional rotations and isospin transformations. The fields $\psi_{A, B}$ defined in this way are labeled by two SU(2) matrices and carry an infinite (reducible) representation of the isospin group and four dimensional rotations. Equivalently, we can define a set of fields $\psi^{j_{1} j_{2} I}$ transforming under finite dimensional irreducible representations. Here, the spin of the field $\psi^{j_{1} j_{2} I}$ ranges, in principle, from $\left|j_{2}-j_{1}\right|$ to $j_{1}+j_{2}$ (I is the isospin); for these fields we define the corresponding Green functions and derive the LSZ formulae to compute the S-matrix, thus mapping a complete relativistic field theory. The S-matrix constraints of locality and unitarity are expected to be satisfied. An heuristic argument could be given along the lines of ref. [9,8], using the Osterwalder-Schrader reconstruction theorem.

The numerical calculation of the saddle point shows that near the singularities we have a region where the chiral symmetry is unbroken, while it is broken away from the singularities. These regions correspond to the unlocalization region, where the topological density is isotropically distributed in Euclidean space-time, and the localization region, where the topological density gets concentrated, signaling the presence of a soliton.

In order to compute the path integrals, we show that, in the localization region, and when the skyrmion is created at rest, the relevant paths can be characterized by (non-covariant) variables $C=B \sqrt{B^{\dagger} A}$, obtained by minimizing the action generated in the unlocalization region.

The covariant propagator is evaluated by using symmetry arguments and inte- 
grating over zero modes in the localization region. The result obtained shows a new symmetry under the transformation $D \rightarrow R D R^{\dagger}$, where $D=\sqrt{B^{\dagger} A}$. This symmetry is seen to be responsible, upon an explicit calculation, for the spin equal to isospin skyrmion spectrum of the model.

Another consequence that shows up, upon fixing the boundary conditions, is the introduction of additional topology, which leads to consider the extension of a map $U: S^{3} \times S^{1} \rightarrow \mathrm{SU}(2)$ to a map $U: S^{3} \times D_{2} \rightarrow \mathrm{SU}(2)$ to study the different possible inequivalent quantizations of the $\mathrm{SU}(2)$-Skyrme model [2]. There are two possibilities, one of them corresponding to the baryonic spectrum composed by half-integer spin (equal to isospin) particles, which are seen to satisfy the spin-statistics theorem.

Finally, we think that our method also provides a formal base for the work in ref. [7] where a skyrmion field theory is advocated (together with an improved approximation scheme) to solve the Yukawa problem, that is, reproducing the pseudovector pion-baryon coupling from skyrmion physics.

\section{Acknowledgement}

We are grateful to D. Mazzitelli, N.N. Scoccola and E.C. Marino for interesting discussions. Collaboration with J.P. Garrahan and C.L. Schat during the first stage of this work is also acknowledged.

\section{A}

In this appendix we summarize a method due to Weinberg [13] to define arbitrary spin field propagators in Minkowski space.

We start by considering states containing one particle (with mass $m$ ) at rest, having spin $j$ and spin projection $\sigma:|(m, 0) ; j \sigma\rangle$ and then we define the states

$$
\left.\left|p ; j \sigma>=\sqrt{\frac{m}{p_{0}}} U\left(L_{p}\right)\right|(m, 0) ; j \sigma\right\rangle,
$$

where $U\left(L_{p}\right)$ is a boost that maps $(m, 0)$ into $p$, which is defined by the $\operatorname{SL}(2, \mathrm{C})$ matrix

$$
L_{p}=\exp (\theta \hat{p} \cdot \vec{\tau})
$$

where $\hat{p}$ is the unit vector $\vec{p} /|\vec{p}|$, and $\sinh (\theta)=|\vec{p}| / m$. Note that $L_{p} L_{p}=$ $\left(p_{0}+\vec{p} . \vec{\tau}\right) / m$. The Lorentz transformations act over the states (A.1) according 
to:

$$
\begin{aligned}
U_{\Lambda}|p ; j \sigma\rangle & =\sqrt{\frac{m}{p_{0}}} U\left(\Lambda L_{p}\right)|(m, 0) ; j \sigma\rangle=\sqrt{\frac{m}{p_{0}}} U\left(L_{\Lambda p}\right) U\left(L_{\Lambda p}^{-1} \Lambda L_{p}\right)|(m, 0) ; j \sigma\rangle \\
& =\sqrt{\frac{(\Lambda p)_{0}}{p_{0}}} D_{\sigma^{\prime} \sigma}^{j}\left(L_{\Lambda p}^{-1} \Lambda L_{p}\right)\left|\Lambda p ; j \sigma^{\prime}\right\rangle .
\end{aligned}
$$

The key point is that $L_{\Lambda p}^{-1} \Lambda L_{p}$ is a rotation and rotations act on the states $|(m, 0) ; j \sigma\rangle$ by means of the $D^{j}$ matrices, as usual.

Particles in the state $|p ; j \sigma\rangle$ are created from the vacuum by operators $a_{p, \sigma}^{\dagger}$ which, in view of eq. (A.3), satisfy:

$$
\begin{aligned}
U_{\Lambda} a_{p, \sigma} U_{\Lambda}^{\dagger} & =\sqrt{\frac{(\Lambda p)_{0}}{p_{0}}} D_{\sigma \sigma^{\prime}}^{j}\left(L_{p}^{-1} \Lambda^{-1} L_{\Lambda p}\right) a_{\Lambda p, \sigma^{\prime}}, \\
U_{\Lambda} a_{p, \sigma}^{\dagger} U_{\Lambda}^{\dagger} & =\sqrt{\frac{(\Lambda p)_{0}}{p_{0}}}\left(C D^{j}\left(L_{p}^{-1} \Lambda^{-1} L_{\Lambda p}\right) C^{-1}\right)_{\sigma \sigma^{\prime}} a_{\Lambda p, \sigma^{\prime}}^{\dagger}
\end{aligned}
$$

Using the operators $a, a^{\dagger}$, and the corresponding operators $b, b^{\dagger}$ for the antiparticles, fields in any finite dimensional representation $\left(j_{1}, j_{2}\right)$ of the Lorentz group (with $\left|j_{2}-j_{1}\right|<j<\left|j_{1}+j_{2}\right|$ ) can be constructed through the formulae

$$
\begin{aligned}
\psi_{m_{1} m_{2}}^{j_{1} j_{2}}= & \int \frac{d^{3} p}{(2 \pi)^{3 / 2}} \frac{1}{\sqrt{2 \omega_{p}}} \mathcal{D}_{m_{1} m_{1}^{\prime}}^{j_{1}}\left(L_{p}\right) \overline{\mathcal{D}}_{m_{2} m_{2}^{\prime}}^{j_{2}}\left(L_{p}\right) \\
& C_{\sigma \sigma^{\prime}}\left(\begin{array}{ccc}
j_{1} & j_{2} & j \\
m_{1}^{\prime} & m_{2}^{\prime} & \sigma^{\prime}
\end{array}\right)\left[a_{p, \sigma} \mathrm{e}^{-\mathrm{ipx}}+(-)^{2 \mathrm{j}_{2}} \mathrm{C}_{\sigma \sigma^{\prime \prime}}^{-1} \mathrm{~b}_{\mathrm{p}, \sigma^{\prime \prime}}^{\dagger} \mathrm{e}^{\mathrm{ipx}}\right], \\
\bar{\psi}_{m_{1} m_{2}}^{j_{1} j_{2}}= & C_{m_{1}^{\prime} m_{1}} C_{m_{2}^{\prime} m_{2}}\left(\psi_{m_{1}^{\prime} m_{2}^{\prime}}^{j_{1} j_{2}}\right)^{\dagger} .
\end{aligned}
$$

The sign $(-)^{2 j_{2}}$ is needed for the $\psi$ and $\psi^{\dagger}$ to commute (or anticommute) at space-like separations. At time-like separations they do not commute since they are built with the same operators $a, a^{\dagger}$. This means that the various relativistic fields we can build from the same spin $j$ particle states are not independent; we must choose one of them to represent these particles. The field $\psi$ transforms as

$$
\mathrm{U}_{\Lambda} \psi_{\mathrm{m}_{1} \mathrm{~m}_{2}}^{\mathrm{j}_{1} \mathrm{j}_{2}}(\mathrm{x}) \mathrm{U}_{\Lambda}^{\dagger}=\mathcal{D}_{\mathrm{m}_{1} \mathrm{~m}_{1}^{\prime}}^{\mathrm{j}_{1}}\left(\Lambda^{-1}\right) \overline{\mathcal{D}}_{\mathrm{m}_{2} \mathrm{~m}_{2}^{\prime}}^{\mathrm{j}_{2}}\left(\Lambda^{-1}\right) \psi_{\mathrm{m}_{1}^{\prime} \mathrm{m}_{2}^{\prime}}^{\mathrm{j}_{1} \mathrm{j}_{2}}(\Lambda \mathrm{x})
$$

while $\bar{\psi}$ obeys a similar relation but with $j_{1}, j_{2}$ interchanged, as corresponds to the adjoint operator. Now we can readily evaluate the propagator

$$
\left\langle 0\left|T\left\{\psi(x)_{m_{1} m_{2}}^{j_{1} j_{2}} \bar{\psi}(y)_{m_{3} m_{4}}^{j_{3} j_{4}}\right\}\right| 0\right\rangle= \begin{cases}\left\langle 0\left|\psi(x)_{m_{1} m_{2}}^{j_{1} j_{2}} \bar{\psi}(y)_{m_{3} m_{4}}^{j_{3} j_{4}}\right| 0\right\rangle & \text { if } x_{0}>y_{0} \\ (-)^{2 j}\left\langle 0\left|\bar{\psi}(y)_{m_{3} m_{4}}^{j_{3} j_{4}} \psi(x)_{m_{1} m_{2}}^{j_{1} j_{2}}\right| 0\right\rangle & \text { if } y_{0}>x_{0}\end{cases}
$$


giving

$$
\begin{aligned}
& \int \frac{d^{3} p}{(2 \pi)^{3} 2 \omega_{p}} \mathcal{D}_{m_{1} m_{1}^{\prime}}^{j_{1}}\left(L_{p}\right) \overline{\mathcal{D}}_{m_{2} m_{2}^{\prime}}^{j_{2}}\left(L_{p}\right) \overline{\mathcal{D}}_{m_{4} m_{4}^{\prime}}^{j_{3}}\left(L_{p}\right) \mathcal{D}_{m_{3} m_{3}^{\prime}}^{j_{4}}\left(L_{p}\right)\left(\begin{array}{ccc}
j_{1} & j_{2} & j \\
m_{1}^{\prime} & m_{2}^{\prime} & \sigma
\end{array}\right) C_{\sigma \sigma^{\prime}} \\
& \left(\begin{array}{ccc}
j_{3} & j_{4} & j \\
m_{3}^{\prime} & m_{4}^{\prime} & \sigma^{\prime}
\end{array}\right)\left[\theta\left(x_{0}-y_{0}\right) \mathrm{e}^{-\mathrm{ip}(\mathrm{x}-\mathrm{y})}+(-)^{\left(2 \mathrm{j}_{2}+2 \mathrm{j}_{4}\right)} \theta\left(\mathrm{y}_{0}-\mathrm{x}_{0}\right) \mathrm{e}^{\mathrm{ip}(\mathrm{x}-\mathrm{y})}\right]
\end{aligned}
$$

For a boost $\overline{\mathcal{D}}^{j}\left(L_{p}\right)=\mathcal{D}^{j}\left(L_{p}^{-1}\right)$; then, using the property of the $3 j$ symbols:

$$
\mathcal{D}_{m_{1} m_{1}^{\prime}}^{j_{1}}\left(L_{p}^{-1}\right)\left(\begin{array}{ccc}
j_{1} & j_{2} & j_{3} \\
m_{1}^{\prime} & m_{2} & m_{3}
\end{array}\right)=\mathcal{D}_{m_{2} m_{2}^{\prime}}^{j_{2}}\left(L_{p}\right) \mathcal{D}_{m_{3} m_{3}^{\prime}}^{j_{3}}\left(L_{p}\right)\left(\begin{array}{ccc}
j_{1} & j_{2} & j_{3} \\
m_{1} & m_{2}^{\prime} & m_{3}^{\prime}
\end{array}\right) .
$$

This expression can be transformed into

$$
\begin{aligned}
\int \frac{d^{3} p}{(2 \pi)^{3} 2 \omega_{p}} & \mathcal{D}_{m_{1} m_{1}^{\prime}}^{j_{1}}\left(L_{p} L_{p}\right) \mathcal{D}_{m_{4} m_{4}^{\prime}}^{j_{4}}\left(L_{p} L_{p}\right) C_{\sigma^{\prime} \sigma}\left(\begin{array}{ccc}
j_{1} & j_{2} & j \\
m_{1} & m_{2}^{\prime} & \sigma^{\prime}
\end{array}\right)\left(\begin{array}{ccc}
j_{3} & j_{4} & j \\
m_{3} & m_{4}^{\prime} & \sigma
\end{array}\right) \\
& {\left[\theta\left(x_{0}-y_{0}\right) \mathrm{e}^{-\mathrm{ip}(\mathrm{x}-\mathrm{y})}+(-)^{\left(2 \mathrm{j}_{2}+2 \mathrm{j}_{4}\right)} \theta\left(\mathrm{y}_{0}-\mathrm{x}_{0}\right) \mathrm{e}^{\mathrm{ip}(\mathrm{x}-\mathrm{y})}\right] . }
\end{aligned}
$$

Taking into account that

$$
\mathcal{D}^{1 / 2}\left(L_{p} L_{p}\right)=L_{p} L_{p}=\frac{1}{m}\left(p_{0}+\vec{p} \cdot \vec{\tau}\right)
$$

and that the elements of the matrix $\mathcal{D}^{j}$ are linear combinations of the elements of $\mathcal{D}^{1 / 2} \otimes \ldots \otimes \mathcal{D}^{1 / 2},(2 j$ times $)$, it follows that $\mathcal{D}^{j}$ is a polynomial in $p_{\mu}$ of order $2 j$. In the integral (A.10) we can "take out" the polynomials $\mathcal{D}^{j}\left(\left(p_{0}+\vec{p} \cdot \vec{\tau}\right) / m\right)$, if we replace $p_{\mu}$ by $-\mathrm{i} \partial_{\mu}$. So the final expression for the Minkowski propagator is

$$
\begin{aligned}
& \left\langle 0\left|T\left\{\psi(x)_{m_{1}, m_{2}}^{j_{1}, j_{2}} \bar{\psi}(y)_{m_{3}, m_{4}}^{j_{3}, j_{4}}\right\}\right| 0\right\rangle=C_{\sigma \sigma^{\prime}}\left(\begin{array}{lll}
j_{1} & j_{2} & j \\
m_{1}^{\prime} & m_{2} & \sigma^{\prime}
\end{array}\right)\left(\begin{array}{lll}
j_{3} & j_{4} & j \\
m_{3} & m_{4}^{\prime} & \sigma
\end{array}\right) \\
& \mathcal{D}_{m_{1} m_{1}^{\prime}}^{j_{1}}\left(-\frac{\mathrm{i}}{m}\left(\partial_{0}+\vec{\tau} . \vec{\partial}\right)\right) \mathcal{D}_{m_{4} m_{4}^{\prime}}^{j_{4}}\left(-\frac{\mathrm{i}}{m}\left(\partial_{0}+\vec{\tau} . \vec{\partial}\right)\right) \Delta_{F}(x-y),
\end{aligned}
$$

where we introduced the scalar Feynman propagator $\Delta_{F}$. When extracting the time derivatives we did not applied them to the $\theta$ functions that define the time ordering. Thus, the above propagator is the so called covariant propagator. The non covariant pieces should be automatically cancelled by non covariant vertices in the interaction Hamiltonian when using the canonical formalism (see [13]). In our case, since the path integral used to define the propagator (eq. (16)) is Lorentz invariant, we also expect to obtain this covariant propagator. As the $\mathcal{D}^{j}\left(-\mathrm{i} \sigma^{\mu} \partial_{\mathrm{m}} \mathrm{u}\right)$ are polynomials in $\partial_{\mu}$, this expression is suitable for 
analytic continuation into Euclidean space. Defining the Euclidean propagator as $\Delta_{E}\left(x_{4}, \vec{x}\right)=\Delta_{F}\left(\mathrm{ix}_{4}, \overrightarrow{\mathrm{x}}\right)$ we obtain the analytic continuation

$$
\begin{aligned}
& C_{\sigma \sigma^{\prime}}\left(\begin{array}{lll}
j_{1} & j_{2} & j \\
m_{1}^{\prime} & m_{2} & \sigma^{\prime}
\end{array}\right)\left(\begin{array}{lll}
j_{3} & j_{4} & j \\
m_{3} & m_{4}^{\prime} & \sigma
\end{array}\right) \\
& \mathcal{D}_{m_{1} m_{1}^{\prime}}^{j_{1}}\left(-\frac{1}{m}\left(\partial_{0}+\mathrm{i} \vec{\tau} . \vec{\partial}\right)\right) \mathcal{D}_{\mathrm{m}_{4} \mathrm{~m}_{4}^{\prime}}^{\mathrm{j}_{4}}\left(-\frac{1}{\mathrm{~m}}\left(\partial_{0}+\mathrm{i} \vec{\tau} . \vec{\partial}\right)\right) \Delta_{\mathrm{E}}(\mathrm{x}-\mathrm{y}) .
\end{aligned}
$$

For large distances $\Delta_{E}(x)$ behaves as

$$
\Delta_{E}(x) \simeq_{|x| \rightarrow \infty} \frac{\mathrm{e}^{-\mathrm{m}|\mathrm{x}|}}{\left(\frac{2 \pi}{m}|x|\right)^{3 / 2}}
$$

and to leading order in $1 /(m|x|)$ we can replace $\partial_{\mu}$ by $-m x_{\mu} / x$, giving

$$
\mathcal{D}_{m_{1} m_{1}^{\prime}}^{j_{1}}(X) \mathcal{D}_{m_{4} m_{4}^{\prime}}^{j_{4}}(X) C_{\sigma \sigma^{\prime}}\left(\begin{array}{ccc}
j_{1} & j_{2} & j \\
m_{1}^{\prime} & m_{2} & \sigma^{\prime}
\end{array}\right)\left(\begin{array}{ccc}
j_{3} & j_{4} & j \\
m_{3} & m_{4}^{\prime} & \sigma
\end{array}\right) \frac{\mathrm{e}^{-\mathrm{m}|\mathrm{x}-\mathrm{y}|}}{\left(\frac{2 \pi}{m}|x-y|\right)^{3 / 2}}
$$

where we introduced the matrix $X=x_{4}+\mathrm{i} \overrightarrow{\mathrm{x}} . \vec{\tau}$.

Another useful calculation that can be done with the free fields (A.5) is the LSZ reduction formula. The procedure is well-known [20]. First we must express $a$, $a^{\dagger}, b, b^{\dagger}$ in terms of the fields $\psi$. This is an easy task which gives, for example, the $a^{\dagger}$ operator as:

$$
\begin{aligned}
& a_{p \sigma}^{\dagger(\text { in })}=\frac{2 j+1}{\sqrt{2 \omega_{p}}}(-)^{2 j}\left(\begin{array}{lll}
j_{1} & j_{2} & j \\
m_{1} & m_{2} & \sigma
\end{array}\right) \overline{\mathcal{D}}_{m_{1} m_{1}^{\prime}}^{j_{1}}\left(L_{p}^{-1}\right) \mathcal{D}_{m_{2} m_{2}^{\prime}}^{j_{2}}\left(L_{p}^{-1}\right) \\
& \int \frac{\mathrm{d}^{3} \mathrm{x}}{(2 \pi)^{3 / 2}} \mathrm{e}^{\mathrm{i} \overrightarrow{\mathrm{p}} \overrightarrow{\mathrm{x}}} \mathrm{e}^{-\mathrm{i} \omega_{\mathrm{p}} \mathrm{t}}\left(\omega_{\mathrm{p}} \bar{\psi}_{\mathrm{m}_{1}^{\prime} \mathrm{m}_{2}^{\prime}}^{(\mathrm{in})}-\mathrm{i} \partial_{\mathrm{t}} \bar{\psi}_{\mathrm{m}_{1}^{\prime} \mathrm{m}_{2}^{\prime}}^{(\mathrm{in})}\right) \text {. }
\end{aligned}
$$

The LSZ reduction proceeds now by using the asymptotic condition for the interacting field

$$
\langle f|\psi(t)| i\rangle \stackrel{t \rightarrow-\infty}{\longrightarrow} Z^{-1 / 2}\left\langle f\left|\psi^{(\mathrm{in})}\right| i\right\rangle,
$$

to replace $\psi^{(\text {in) }}$ by $\psi$ in the formula for $a_{p, \sigma}^{\dagger}$. The renormalization factor $Z$ will absorb the factor $\exp \left(-C_{\epsilon}\right)$ introduced in eq. (16). Then, using the relation

$$
\lim _{t \rightarrow-\infty} f(t)=-\int_{-\infty}^{+\infty} \partial_{t} f(t) d t+\lim _{\mathrm{t} \rightarrow+\infty} \mathrm{f}(\mathrm{t})
$$

we arrive at the reduction formula. The result for $a^{\dagger}$ as well as for the other 
operators is

$$
\begin{aligned}
& \left\langle\text { out }\left|\mathrm{a}_{\mathrm{p} \sigma}^{\dagger(\mathrm{in})}\right| \text { in }\right\rangle=\left\langle\text { out }\left|\mathrm{a}_{\mathrm{p} \sigma}^{\dagger(\text { out })}\right| \text { in }\right\rangle+\mathrm{iZ}^{1 / 2} \frac{2 \mathrm{j}+1}{\sqrt{2 \omega_{\mathrm{p}}}}(-)^{2 \mathrm{j}}\left(\begin{array}{ccc}
j_{1} & j_{2} & j \\
m_{1} & m_{2} & \sigma
\end{array}\right) \overline{\mathcal{D}}_{\mathrm{m}_{1} \mathrm{~m}_{1}^{\prime}}^{\mathrm{j}_{1}}\left(\mathrm{~L}_{\mathrm{p}}^{-1}\right) \\
& \mathcal{D}_{m_{2} m_{2}^{\prime}}^{j_{2}}\left(L_{p}^{-1}\right) \int \frac{\mathrm{d}^{4} \mathrm{x}}{(2 \pi)^{3 / 2}} \mathrm{e}^{-\mathrm{ipx}}\left(\square+\mathrm{m}^{2}\right)\left\langle\text { out }\left|\bar{\psi}_{\mathrm{m}_{1}^{\prime} \mathrm{m}_{2}^{\prime}}^{\mathrm{j}_{1} \mathrm{j}_{2}}\right| \text { in }\right\rangle \\
& =\text { disc. }+\mathrm{i} Z^{1 / 2} \int \mathrm{d}^{4} \mathrm{xe}^{-\mathrm{ipx}} \overline{\mathrm{u}}_{\mathrm{m}_{1} \mathrm{~m}_{2}}^{\mathrm{j}_{\mathrm{j}} \mathrm{j}_{2}}(\mathrm{p} \sigma)\left(\square+\mathrm{m}^{2}\right)\left\langle\text { out }\left|\bar{\psi}_{\mathrm{m}_{1} \mathrm{~m}_{2}}^{\mathrm{j}_{\mathrm{j} \mathrm{j}_{2}}}\right| \mathrm{in}\right\rangle, \\
& \left\langle\text { out }\left|\mathrm{b}_{\mathrm{p} \sigma}^{\dagger(\text { in })}\right| \text { in }\right\rangle=\left\langle\text { out }\left|\mathrm{b}_{\mathrm{p} \sigma}^{\dagger(\text { out })}\right| \text { in }\right\rangle+\mathrm{iZ}^{1 / 2} \frac{2 \mathrm{j}+1}{\sqrt{2 \omega_{\mathrm{p}}}}(-)^{2 \mathrm{j}_{1}}\left(\begin{array}{ccc}
j_{1} & j_{2} & j \\
m_{1} & m_{2} & \sigma
\end{array}\right) \mathcal{D}_{\mathrm{m}_{1} \mathrm{~m}_{1}^{\prime}}^{\mathrm{j}_{1}}\left(\mathrm{~L}_{\mathrm{p}}^{-1}\right) \\
& \overline{\mathcal{D}}_{m_{2} m_{2}^{\prime}}^{j_{2}}\left(L_{p}^{-1}\right) \int \frac{\mathrm{d}^{4} \mathrm{x}}{(2 \pi)^{3 / 2}} \mathrm{e}^{-\mathrm{ipx}}\left(\square+\mathrm{m}^{2}\right)\left\langle\text { out }\left|\psi_{\mathrm{m}_{1}^{\prime} \mathrm{m}_{2}^{\prime}}^{\mathrm{j}_{1} \mathrm{j}_{2}}\right| \text { in }\right\rangle \\
& =\text { disc. }+\mathrm{iZ}^{1 / 2} \int \mathrm{d}^{4} \mathrm{xe}^{-\mathrm{ipx}} \mathrm{v}_{\mathrm{m}_{1} \mathrm{~m}_{2}}^{\mathrm{j}_{1} \mathrm{j}_{2}}(\mathrm{p} \sigma)\left(\square+\mathrm{m}^{2}\right)\left\langle\text { out }\left|\psi_{\mathrm{m}_{1} \mathrm{~m}_{2}}^{\mathrm{j}_{1} \mathrm{j}_{2}}\right| \mathrm{in}\right\rangle, \\
& \left\langle\text { out }\left|\mathrm{a}_{\mathrm{p} \sigma}^{(\text {out })}\right| \text { in }\right\rangle=\left\langle\text { out }\left|\mathrm{a}_{\mathrm{p} \sigma}^{(\mathrm{in})}\right| \text { in }\right\rangle+\mathrm{iZ} \mathrm{Z}^{1 / 2} \frac{2 \mathrm{j}+1}{\sqrt{2 \omega_{\mathrm{p}}}}(-)^{2 \mathrm{j}} \mathrm{C}_{\sigma \sigma^{\prime}}\left(\begin{array}{ccc}
j_{1} & j_{2} & j \\
m_{1} & m_{2} & \sigma^{\prime}
\end{array}\right) \mathcal{D}_{\mathrm{m}_{1} \mathrm{~m}_{1}^{\prime}}^{\mathrm{j}_{1}}\left(\mathrm{~L}_{\mathrm{p}}^{-1}\right) \\
& \overline{\mathcal{D}}_{m_{2} m_{2}^{\prime}}^{j_{2}}\left(L_{p}^{-1}\right) \int \frac{\mathrm{d}^{4} \mathrm{x}}{(2 \pi)^{3 / 2}} \mathrm{e}^{\mathrm{ipx}}\left(\square+\mathrm{m}^{2}\right)\left\langle\text { out }\left|\psi_{\mathrm{m}_{1}^{\prime} \mathrm{m}_{2}^{\prime}}^{\mathrm{j}_{1} \mathrm{j}_{2}}\right| \text { in }\right\rangle \\
& =\text { disc. }+\mathrm{iZ}^{1 / 2} \int \mathrm{d}^{4} \mathrm{xe}^{\mathrm{ipx}} \mathrm{u}_{\mathrm{m}_{1} \mathrm{~m}_{2}}^{\mathrm{j}_{1} \mathrm{j}_{2}}(\mathrm{p} \sigma)\left(\square+\mathrm{m}^{2}\right)\left\langle\text { out }\left|\psi_{\mathrm{m}_{1} \mathrm{~m}_{2}}^{\mathrm{j}_{1} \mathrm{j}_{2}}\right| \text { in }\right\rangle, \\
& \left\langle\text { out }\left|\mathrm{b}_{\mathrm{p} \sigma}^{(\text {out })}\right| \text { in }\right\rangle=\left\langle\text { out }\left|\mathrm{b}_{\mathrm{p} \sigma}^{(\mathrm{in})}\right| \mathrm{in}\right\rangle+\mathrm{iZ} \mathrm{Z}^{1 / 2} \frac{2 \mathrm{j}+1}{\sqrt{2 \omega_{\mathrm{p}}}}(-)^{2 \mathrm{j}_{1}} \mathrm{C}_{\sigma \sigma^{\prime}}\left(\begin{array}{ccc}
j_{1} & j_{2} & j \\
m_{1} & m_{2} & \sigma^{\prime}
\end{array}\right) \overline{\mathcal{D}}_{\mathrm{m}_{1} \mathrm{~m}_{1}^{\prime}}^{\mathrm{j}_{1}}\left(\mathrm{~L}_{\mathrm{p}}^{-1}\right) \\
& \mathcal{D}_{m_{2} m_{2}^{\prime}}^{j_{2}}\left(L_{p}^{-1}\right) \int \frac{\mathrm{d}^{4} \mathrm{x}}{(2 \pi)^{3 / 2}} \mathrm{e}^{\mathrm{ipx}}\left(\square+\mathrm{m}^{2}\right)\left\langle\text { out }\left|\bar{\psi}_{\mathrm{m}_{1}^{\prime} \mathrm{m}_{2}^{\prime}}^{\mathrm{j}_{\mathrm{j}}}\right| \text { in }\right\rangle \\
& =\text { disc. }+\mathrm{i} Z^{1 / 2} \int \mathrm{d}^{4} \mathrm{xe}^{\mathrm{ipx}} \overline{\mathrm{v}}_{\mathrm{m}_{1} \mathrm{~m}_{2}}^{\mathrm{j}_{1} \mathrm{j}_{2}}(\mathrm{p} \sigma)\left(\square+\mathrm{m}^{2}\right)\left\langle\text { out }\left|\bar{\psi}_{\mathrm{m}_{1} \mathrm{~m}_{2}}^{\mathrm{j}_{1} \mathrm{j}_{2}}\right| \text { in }\right\rangle,(\text { A. } 19)
\end{aligned}
$$

where for brevity we defined $u, \bar{u}, v, \bar{v}$ which can be interpreted as wave functions of incoming and outgoing particles. These formulae allow us to extract the scattering amplitudes from the Green functions we defined.

\section{References}

[1] E. Witten, Nucl. Phys. B160, 57 (1979); G.S. Adkins, C. Nappi and E. Witten, Nucl. Phys. B228, 522 (1983).

[2] E. Witten, Nucl. Phys. B223, 422 (1983); B223 433 (1983). 
[3] T.H.R. Skyrme, Proc. R. Soc. London 260, 127 (1961); Nucl. Phys. 31, 556 (1962).

[4] B. Moussallam, Ann. Phys. 225, 264 (1993).

[5] J. Gasser and H. Leutwyler, Ann. Phys. 158 142, (1984).

[6] E. Witten, Nucl. Phys. B223, 57 (1979).

[7] N . Dorey, J. Hughes and M.P. Mattis, Phys. Rev. D 505816 (1994) (hep$\mathrm{ph} / 9404274)$.

[8] J. Fröhlich and P.A. Marchetti, Nucl. Phys. B335 1 (1990).

[9] J. Fröhlich and P.A. Marchetti, Comm. Math. Phys. 112, 343 (1987), 116127 (1988), 121177 (1989).

[10] E.C. Marino, J.A. Swieca, Nucl. Phys. B 170, 175 (1980), E.C. Marino, B. Schroer, J.A. Swieca, Nucl. Phys. B 200, 473 (1982).

[11] G. 't Hooft, Nucl. Phys B138 1 (1978).

[12] I. Zahed and G.E. Brown, Phys. Rep. 142 (1986)

[13] S. Weinberg, Phys. Rev. 5B, 1318 (1964).

[14] N.S. Manton and P.J. Ruback, Phys.Lett.B 181, 137 (1986).

[15] A.R. Edmonds, Angular Momentum in Quantum Mechanics (Princeton University Press, Princeton, 1960).

[16] L.S. Schulman, Techniques and applications of Path Integration (John Wiley and Sons, New York, 1981).

[17] R.P. Feynman and M. Gell-Mann, Phys.Rev. 109, 193 (1958).

[18] D.I. Dyakonov, V. Yu. Petrov and P.B. Pobylitsa, Phys. Lett. B205, 372 (1988).

[19] A. Hayashi, S. Saito and M. Uehara, Phys. Lett. B246, 15 (1990).

[20] C. Itzykson and J.B. Zuber, Quantum Field Theory (Mc Graw-Hill, New York, 1980). 